\title{
Integrated transcriptomic and proteomic analysis of Tritipyrum provides insights into the molecular basis of salt tolerance
}

\author{
Rui Yang Equal first author, ${ }^{1}$, Zhifen Yang Equal first author, ${ }^{1}$, Ze Peng ${ }^{1}, \mathbf{f}$ ang h $\mathbf{~ e ~}{ }^{1}$, Luxi Shi $^{1}$, Yabing Dong ${ }^{1}$, Mingjian Ren ${ }^{1,2}$, \\ Qingqin Zhang ${ }^{1}$, Guangdong Geng ${ }^{\text {Corresp., }}{ }^{\text {, Suqin Zhang }}{ }^{\text {Corresp. 1, } 2}$ \\ ${ }^{1}$ Guizhou University, Guiyang, China \\ 2 Guizhou Subcenter of National Wheat Improvement Center, Guiyang, China \\ Corresponding Authors: Guangdong Geng, Suqin Zhang \\ Email address: gdgeng@gzu.edu.cn, sqzhang1@gzu.edu.cn
}

Background. Soil salinity is a major environmental stress that restricts crop growth and yield. Methods. Here, crucial proteins and biological pathways were investigated under salt-stress and recovery conditions in Tritipyrum 'Y1805' using the data-independent acquisition proteomics techniques to explore its salt-tolerance mechanism. Results. In total, 44 and 102 differentially expressed proteins (DEPs) were identified in ' $Y 1805$ ' under salt-stress and recovery conditions, respectively. A proteome-transcriptome-associated analysis revealed that the expression patterns of 13 and 25 DEPs were the same under salt-stress and recovery conditions, respectively. 'Response to stimulus', 'antioxidant activity', 'carbohydrate metabolism', 'amino acid metabolism', 'signal transduction', 'transport and catabolism' and 'biosynthesis of other secondary metabolites' were present under both conditions in 'Y1805'. In addition, 'energy metabolism' and 'lipid metabolism' were recovery-specific pathways, while 'antioxidant activity', and 'molecular function regulator' under salt-stress conditions, and 'virion' and 'virion part' during recovery, were 'Y1805'-specific compared with the salt-sensitive wheat 'Chinese Spring'. 'Y1805' contained 83 specific DEPs related to salt-stress responses. The strong salt tolerance of 'Y1805' could be attributed to the strengthened cell walls, reactive oxygen species scavenging, osmoregulation, phytohormone regulation, transient growth arrest, enhanced respiration, transcriptional regulation and error information processing. These data will facilitate an understanding of the molecular mechanisms of salt tolerance and aid in the breeding of salt-tolerant wheat. 
1 Integrated transcriptomic and proteomic analysis of

2 Tritipyrum provides insights into the molecular basis

3 of salt tolerance

4

5 Rui Yang ${ }^{1 *}$, Zhifen Yang ${ }^{1 *}$, Ze Peng ${ }^{1}$, fang he ${ }^{1,2}$, Luxi Shi ${ }^{1}$, Yabing Dong ${ }^{1}$, Mingjian Ren ${ }^{1,2}$,

6 Qingqin Zhang ${ }^{1}$, Guangdong Geng ${ }^{1}$ and Suqin Zhang ${ }^{1,2}$

7

$8 \quad{ }^{1}$ College of Agriculture, Guizhou University, Guiyang, Guizhou 550025

$9{ }^{2}$ Guizhou Subcenter of National Wheat Improvement Center, Guiyang, Guizhou 550025

11 Corresponding authors

12 Suqin Zhang, sqzhang1@gzu.edu.cn

13 Guangdong Geng, gdgeng@gzu.edu.cn

14

$15 *$ These authors contributed equally to this work. 
16 ABSTRACT

17 Background. Soil salinity is a major environmental stress that restricts crop growth and yield.

Methods. Here, crucial proteins and biological pathways were investigated under salt-stress and recovery conditions in Tritipyrum 'Y1805' using the data-independent acquisition proteomics techniques to explore its salt-tolerance mechanism.

Results. In total, 44 and 102 differentially expressed proteins (DEPs) were identified in 'Y1805'

under salt-stress and recovery conditions, respectively. A proteome-transcriptome-associated

analysis revealed that the expression patterns of 13 and 25 DEPs were the same under salt-stress

and recovery conditions, respectively. 'Response to stimulus', 'antioxidant activity',

'carbohydrate metabolism', 'amino acid metabolism', 'signal transduction', 'transport and

catabolism' and 'biosynthesis of other secondary metabolites' were present under both

conditions in 'Y1805'. In addition, 'energy metabolism' and 'lipid metabolism' were recovery-

specific pathways, while 'antioxidant activity', and 'molecular function regulator' under salt-

stress conditions, and 'virion' and 'virion part' during recovery, were 'Y1805'-specific

compared with the salt-sensitive wheat 'Chinese Spring'. 'Y1805' contained 83 specific DEPs

related to salt-stress responses. The strong salt tolerance of 'Y1805' could be attributed to the

strengthened cell walls, reactive oxygen species scavenging, osmoregulation, phytohormone

regulation, transient growth arrest, enhanced respiration, transcriptional regulation and error

information processing. These data will facilitate an understanding of the molecular mechanisms

of salt tolerance and aid in the breeding of salt-tolerant wheat. 


\section{INTRODUCTION}

Wheat (Triticum aestivum) is the most important food crop in the world. Its total area, total output and total trade volume all rank first among food crops, and it provides $19 \%$ of available calories for humans (Yan et al., 2020). It is predicted that by the middle of the $21^{\text {st }}$ century, increasing salinization will lead to the loss of $50 \%$ of the world's arable land (Munns \& Tester, 2008; Goyal et al., 2016). Salt stress is a major challenge for wheat production because it has devastating effects on crop yield. Salt stress can occur at any time during the life cycle of wheat (Setter et al., 2016; Ghobadi ME, Ghobadi $M \&$ Zebarjadi, 2017). Therefore, finding ways to improve crop tolerance to salt stress is essential to improve wheat productivity and achieve food security.

Salt accumulation in the soil causes osmotic imbalance, ion toxicity, nutritional disorders, normal metabolic disruption, reactive oxygen species (ROS) accumulation and oxidative stressrelated injury (Tang et al., 2015; Ma et al., 2020). Plants have evolved complex cellular, physiological and molecular mechanisms for responding and adapting to environmental cues, including salt stress (Chinnusamy, Jagendorf \& Zhu, 2005; Deinlein et al., 2014; Fahad et al., 2015; Kumar et al., 2017; Mahajan et al., 2017; Mahajan et al., 2020). Physiological studies have demonstrated that osmotic adjustment, ion homeostasis, antioxidant systems, hormonal regulation, nutrient uptake and signaling play important roles in salt tolerance (Zhao et al., 2020). In addition, dramatic alterations in gene expression, which lead to changes in protein profiles, are closely related to these processes (Du et al., 2010; Sobhanian et al., 2011).

Proteins are directly involved in shaping plant phenotypes through either structural or 
58

59

60

regulatory functions related to the formation of the plant epigenome, transcriptome and metabolome. Quantitative proteomics has become a powerful tool in the identification of proteins and mechanisms involved in salt responses and tolerance. The enhanced salinity tolerance of durum wheat appears to be governed by a higher capacity for osmotic homeostasis, a more efficient defense by late embryogenesis abundant (LEA), redox and pathogenesis-related proteins, and an improved protection from mechanical stress by increased cell wall lignification (Capriotti et al., 2014). The enhanced salinity tolerance of the salt-tolerant barley 'DH187' results mainly from the increased activity levels of signal-transduction mechanisms, which eventually leads to the accumulation of stress-protective proteins and cell-wall structural changes (Mostek et al., 2015). A total of 514 and 770 protein spots were reported in the most salt-tolerant and salt-sensitive rice cultivars, respectively. The differentially expressed proteins (DEPs) are associated with major metabolic pathways, including photosynthesis, energy metabolism, amino acid metabolism and nitrogen assimilation, as well as stress and signaling pathways (Frukh et al., 2020). In salt-stressed maize ' 8723 ', the DEPs are primarily associated with phenylpropanoid biosynthesis, starch and sucrose metabolism, and mitogen-activated protein kinase signaling pathways (Chen et al., 2019). It is important to identify valuable key genes and proteins from the large amounts of data obtained by transcriptome sequencing and proteome technologies. In total, the expression levels of 13 and 11 genes in salt-tolerant and -sensitive barley genotypes are consistent after salt stress at the transcriptome and proteome levels, respectively (Lai et al., 2020). In salt-tolerant sweet potato, eight differentially expressed genes (DEGs) display the same pattern under salt-stress conditions at the transcriptome and proteome levels (Meng et al., 2020). 
SWATH-MS is a recently introduced technique consisting of a data-independent acquisition (DIA) and a targeted data analysis strategy that aims to maintain the favorable quantitative characteristics (accuracy, sensitivity and selectivity) achieved in targeted proteomics but on the scale of thousands of proteins. The E genome species (e.g., halophile wheatgrass Thinopyrum elongatum) of the Triticeae are invaluable sources of salt tolerance (Jauhar, 1990; Munns \& Tester, 2008; Margiotta et al., 2020). Tritipyrum derived from the wide crosses of Triticum and Thinopyrum show salt tolerance (King et al., 1997; Yuan \& Tomita, 2015). Currently, there are limited reports on the molecular mechanisms of Tritipyrum salt tolerance; therefore, they remain largely unknown. Here, protein markers and pathways were identified using DIA proteomic and transcriptomic profiling, and these will aid in elucidating the molecular mechanisms involved in the salt tolerance of Tritipyrum. The results provide valuable information for the molecular breeding of improved salt tolerance in wheat and other crops.

\section{MATERIALS AND METHODS}

\section{Plant materials}

'Y1805' is a stable progeny from the wide cross between Triticum aestivum and Thinopyrum elongtum. Salt-tolerant Tritipyrum ('Y1805') and salt-sensitive Triticum aestivum ('Chinese Spring', 'CS') were used in this experiment. These genotypes were chosen on the basis of preliminary experiments. 'Y1805' shows plump kernels, mid-maturity and high complex disease-resistance levels, including immunity and high resistance levels to scab, rust and powdery mildew of wheat.

\section{Plant growth conditions and stress treatments}

The seeds of 'Y1805' and 'CS' were germinated in a growth chamber (relative humidity of 75\% 
101

102

103

104

105

106

107

108

109

110

111

112

113

114

115

116

117

118

119

120

121

and a $20 / 15^{\circ} \mathrm{C}$ light/dark photocycle). The seedlings were sown on a floater board in $1 / 2$

Hoagland's solution with a 16-/8-h light/dark cycle, irradiance of $400 \mu \mathrm{mol} \mathrm{m}^{-2} \mathrm{~s}^{-1}$ and the same

temperature and humidity as in the germination chamber. The culture solution was refreshed

every 3 days. On the $14^{\text {th }}$ day (two-leaf stage), salt-stress treatments $(1 / 2$ Hoagland's solution

supplemented with $250 \mathrm{mM} \mathrm{NaCl}$ ) were started. A 250-mM NaCl concentration was used as a

result of preliminary gradient tests. The $\mathrm{pH}$ of the culture solution was 6.0 . The first wheat root

and shoot samples of a uniform size were selected at $5 \mathrm{~h}$ (T1 stage) after exposure to salt stress.

The materials were recovered (in 1/2 Hoagland's solution without $\mathrm{NaCl}$ ) after $24 \mathrm{~h}$ of salt stress.

The second and third samplings were performed at $1 \mathrm{~h}$ (T2 stage) and $24 \mathrm{~h}$ (T3 stage) after

recovery, respectively. These sampling stages were chosen on the basis of the phenotypic and

physiological data. Normal (1/2 Hoagland's solution without $\mathrm{NaCl}$ ) control cultured

materials, CK1, CK2 and CK3, were used as parallel controls for T1, T2 and T3,

respectively. The root samples of the $\mathrm{T} 1$ and $\mathrm{T} 2$ stages were immediately frozen in

liquid nitrogen after sampling and stored at $-80^{\circ} \mathrm{C}$ for the transcriptomic analysis,

proteomic analysis, and confirmational qRT-PCR. The samples at the T3 stage were

only used for growth analysis. Three biological replications and two technical replications

were employed, and at least 10 seedlings were mixed per replicate.

\section{Protein analysis}

\section{Protein extraction and DIA-MS/MS analysis}

Total proteins of wheat roots were extracted using the phenol method with slight modifications

(Isaacson et al., 2006). Briefly, $0.5 \mathrm{~g}$ of wheat roots were ground into a fine powder in a lysis

PeerJ reviewing PDF | (2021:05:60891:2:0:NEW 31 Oct 2021) 
122

123

124

125

126

127

128

129

130

131

132

133

134

135

buffer containing $8 \mathrm{M}$ urea, $400 \mathrm{mM}$ ammonium bicarbonate, $10 \mathrm{mM}$ dithiothreitol and a protease inhibitor cocktail (Roche, Basel, Switzerland). Then, $2 \times$ volume of phenol saturated with Tris- $\mathrm{HCl}(\mathrm{pH} 7.5)$ was added, and centrifuged at $25,000 \mathrm{~g}$ for $15 \mathrm{~min}$ at $4{ }^{\circ} \mathrm{C}$. The upper phenolic phase was collected, and then, $5 \times$ volume of precooled precipitation solution, containing $0.1 \mathrm{M}$ ammonium acetate in methanol and $10 \mathrm{mM}$ dithiothreitol, was added to the protein mixture. Each sample was maintained for $2 \mathrm{~h}$ at $-20^{\circ} \mathrm{C}$. Then, the samples were centrifuged at $25,000 \mathrm{~g}$ for $15 \mathrm{~min}$ at $4{ }^{\circ} \mathrm{C}$, and the supernatant was removed. The pellets were further washed with precooled acetone with centrifugation at 20,400 g for $40 \mathrm{~min}$. Then, trypsin [protein:trypsin $=40: 1(\mathrm{w} / \mathrm{w})]$ was added for enzymolysis, followed by desalination, vacuum drying, and redissolution. Protein concentrations were measured by using Nanodrop ND-1000 (Thermo Fisher Scientific, San Jose, CA, USA).

The peptide samples were separated using an UltiMate 3000 UHPLC (Thermo Fisher

Scientific, San Jose, CA, USA), ionized by a nanoESI source and then put into a Q-Ex Active HF tandem mass spectrometer (Thermo Fisher Scientific, San Jose, CA, USA) for DIA mode detection. The spectral library was constructed using the DIA of targeted samples. Spectronaut was used to effectively deconvolute, accurately identify and quantitatively analyze the data (Bernhardt et al., 2014).

\section{DEP identification and bioinformatics analysis}

The peak area of an ion pair was extracted using Spectronaut. Then, the Msstats software package was used to complete the error correction and normalization steps (Choi., et al., 2014). Two conditions, fold change $\geq 2$ and false discovery rate $<0.05$, were used as the DEP criteria. 
143 The Gene Ontology (GO) and Kyoto Encyclopedia of Genes and Genomes (KEGG) enrichment

144 analyses, protein-protein interaction (PPI) and subcellular localization analysis were performed

145 for these DEPs.

146 Proteome-transcriptome-associated analysis

147 The basis of proteome-transcriptome association analysis is the central principle. Correlation

148 analyses of mRNAs and proteins having the same change trend were carried out, including

149 expression correlation analyses and metabolic pathway map integration analyses.

150

151

152

\section{The quantitative RT-PCR (qRT-PCR) analysis}

15 candidate proteins were verified by qRT-PCR (Table S1). Total RNA from the same samples used in the proteome analysis were reverse-transcribed using Power SYBR ${ }^{\circ}$ Green PCR Master Mix (Applied Biosystems, Foster, CA, USA). qRT-PCR amplification was performed on an ABI StepOne Real-Time PCR System. The relative expression levels were calculated in accordance with the $2^{-\Delta \Delta \mathrm{Ct}}$ method, with three biological replications and three technical replications (Depuydt \& Hardtke, 2011), and $\beta$-actin and $18 S R N A$ were used as the internal control.

\section{Statistical analyses}

The data were analyzed using SPSS statistics 19.0 (IBM Corp., Armonk, NY). Statistical differences between means were determined by Duncan's multiple range test at a significance level of $p<0.05$ after displaying a significant effect during an ANOVA. Pearson's correlation analysis of binary variables was performed, and two variables were considered significantly correlated at the $p<0.05$ level.

\section{RESULTS}

Response of plant growth to salt stress and recovery in two wheat 
165

166

167

168

169

170

171

172

173

174

175

176

177

178

179

180

181

182

183

184

185

\section{varieties}

The plant length and dry weight of salt-tolerant Tritipyrum 'Y1805' did not change significantly

at any of the three stages compared with the corresponding controls (Fig. 1). Additionally,

'Y1805' maintained normal growth under salt-stress conditions. The plant length of salt-sensitive

'CS' was significantly $(p<0.05)$ less than that of the control at the T2 stage. At the T3 stage, the plant length and dry weight of 'CS' decreased significantly $(p<0.05)$ by $6.37 \%$ and $15.69 \%$, respectively, compared with the controls. Thus, 'Y1805' showed a strong salt tolerance under salt-stress conditions and a rapid recovery capability after the salt stress was removed.

\section{Quantitative analysis of DEPs}

The DEP quantitative analysis was carried out under salt-stress and recovery conditions in 'Y1805' and 'CS'. Under salt-stress conditions, 44 DEPs were identified in 'Y1805' ('Y1805' T1), among which 21 were upregulated and 23 downregulated (Fig. 2A). For 'CS' ('CS' T1), 69 (29 upregulated and 40 downregulated) DEPs were identified. Among these DEPs, 21 (10 upregulated and 11 downregulated) proteins were common to both genotypes and showed the same expression patterns (Fig. 2B, Table S2), indicating that they represented the core genes associated with responses to salt stress. 'Y1805' had 23 specific DEPs, including 11 upregulated and 12 downregulated DEPs, which should play key roles in its strong salt-tolerance.

During the recovery process, 102 (66 upregulated and 36 downregulated) DEPs were identified in 'Y1805' ('Y1805' T2). In 'CS' ('CS' T2), 525 DEPs were detected. A total of 29 DEPs appeared in both varieties (Table S3), with 96.55\% displaying the same expression 
186

187

188

189

190

191

192

193

202

203

204

205

206

207

patterns. 'Y1805' had 73 (48 upregulated and 25 downregulated) specific DEPs that might contribute to its fast recovery after salt stress.

In total, 14 DEPs were identified under both salt-stress and recovery conditions in 'Y1805'

(Table S4). Therefore, these DEPs might simultaneously participate in the responses to salt stress and recovery. Moreover, these DEPs showed the same expression patterns at the two stages, with 10 DEPs being upregulated and 4 DEPs being downregulated compared with the respective corresponding controls. More specific DEPs were identified after recovery than under salt-stress conditions in 'Y1805'. Thus, salt stress and recovery regulated different types of proteins, allowing plants to adapt to environmental stress or growth restoration, respectively. 15 DEPs closely related to salt stress were selected from 'Y1805' for the qRT-PCR analysis (Table S1). The correlation coefficient of the relative expression levels between DEPs and qRTPCR was significant $\left(\mathrm{r}=0.802, p=1.97 \mathrm{E}^{-04}\right)$. The up- or downregulation of the proteins, as determined by the proteomics analysis, was corroborated by the qRT-PCR experiment (Fig. 2C). These data demonstrated that the proteomics accurately reflected the responses of Tritipyrum to salt stress and recovery.

\section{Enrichment analysis}

\section{DEP enrichment analysis under salt-stress conditions}

GO and KEGG enrichment analyses of the common DEPs in 'Y1805' and 'CS' were performed under salt-stress conditions (Fig. S1). 'Catalytic activity' (GO:0003824), 'binding' (GO:0005488) (molecular functions), 'cell' (GO:0005623 ), 'cell part' (GO:0044464), 'membrane part' (GO:0044425), ‘membrane’ (GO:0016020), ‘organelle’ (GO:0043226) (cell components), 
208

209

210

211

212

213

214

215

216

217

218

219

220

221

222

223

224

225

226

229

227 DEP enrichment analysis during the recovery process

228 The molecular functions of common DEPs in 'Y1805' and 'CS' were 'binding', 'catalytic

'cellular process' (GO:0009987), 'metabolic process' (GO:0008152) and 'cellular component organization or biogenesis' (GO:0071840) (biological process) were significantly enriched in the GO analysis. The KEGG pathways ‘environmental adaptation' (ko04626), 'global and overview maps' (ko01100), ‘carbohydrate metabolism' (ko00052), ‘amino acid metabolism' (ko00360), 'biosynthesis of other secondary metabolites' (ko00940), 'nucleotide metabolism' (ko00230), 'signal transduction' (ko04011), 'translation' (ko03010) and 'transport and catabolism' (ko04144) were significantly enriched. These GO terms and KEGG pathways had fundamental roles in the responses to salt stress in 'Y1805' and 'CS'.

Plant antioxidant proteins are part of defense systems and play important roles in response to salt stress. 'Antioxidant activity' (GO:0004601) and 'molecular function regulator' (GO:0004867) were two 'Y1805'-specific GO terms (Fig. 3A, Fig. S1, S2). The antioxidative activity was regulated by three peroxidase (POD) superfamily proteins: peroxidase 5 AT1G14550 (TraesCS1B01G096300.1), peroxidase 47 AT4G33420 (TraesCS2B01G616700.1) and peroxidase 50-like AT4G37520 (TraesCS2A01G502100.1) (Table S5). POD plays pivotal roles in the removal of $\mathrm{H}_{2} \mathrm{O}_{2}$. The molecular functional regulatory protein was subtilisin chymotrypsin inhibitor-2A EMT04243 (TraesCS3A01G093900.1). The subcellular localization of 'Y1805'-specific DEPs under salt-stress conditions revealed that most were cytosolic proteins, followed by chloroplastic and then extracellular (Fig. 3C). activity', 'antioxidant activity' and 'transporter activity' (GO:0005215) during the recovery 
230 process (Fig. S3A). In the cell component category, they were mainly 'cell', 'cell part',

231 'organelle' and 'extracellular region' (GO:0005576). In the biological process category, 'cellular

232 process' and 'metabolic process' predominated. The enriched KEGG pathways were 'global and

233 overview maps', 'amino acid metabolism', 'biosynthesis of other secondary metabolites',

234 'carbohydrate metabolism', 'metabolism of other amino acids' (ko00410), 'transport and

235 catabolism', 'signal transduction', 'lipid metabolism' (ko00561) and 'energy metabolism'

236 (ko00920) (Fig. S3B). These GO terms and KEGG pathways had major roles in the growth

237 recovery of 'Y1805' and 'CS' after salt stress.

238 'Y1805' was enriched for two specific pathways, 'virion' (GO:0005634) and 'virion part'

239 (GO:0019013), during the recovery process (Fig. 4A, Fig. S4A). Their DEPs were H/ACA

240 ribonucleoprotein complex subunit 3-like NOP10 (TraesCS6B01G254000.1) (Table S5), which

241 is necessary for ribosomal biogenesis and a part of the complex that catalyzes the

242 pseudouridylation of rRNA. Most 'Y1805'-specific DEPs localized to chloroplasts, followed by

243 cytosol and then nuclei (Fig. 4C).

244

245 DEP interaction analysis

246 PPI analysis was conducted for 'Y1805' DEPs during the salt-stress and recovery stages (Fig. 5).

247 In total, 7 nodal proteins were detected among the 44 DEPs under salt-stress conditions (Table

248 S6). Probable cellulose synthase A catalytic subunit 8 IRX1 (TraesCS2A01G102600.1)

249 interacted with both phenylalanine ammonia-lyase 1 PAL1 (TraesCS2D01G377600.1) and

250 probable cellulose synthase A catalytic subunit 1 CESA1 (TraesCS1B01G136200.1) (Table S5). 
251 PAL1 interacted with arogenate dehydratase/prephenate dehydratase 6 ADT6

252 (TraesCS2A01G292000.1), CESA1 interacted with glucomannan 4-beta-mannosyltransferase 1

CSLA01 (TraesCS6A01G169200.1), and endoglucanase 17 GH9B13 (TraesCS7A01G204500.1) interacted with ethylene-forming enzyme EFE (TraesCS1A01G089500.1). ADT6 and PAL1 are related to lignin synthesis, while IRX1 and CESA1 are involved in cellulose synthesis. The mannose polysaccharide produced by CSLA01 is a cell-wall component. Therefore, PAL1, ADT6, IRX1, CESA1 and CSLA01 were all closely related to the synthesis of the cell wall, indicating that cell-wall integrity under salt-stress conditions was crucial for salt tolerance.

In total, 32 nodal proteins were found among the 102 DEPs identified during the recovery process (Table S6). 1-(5-phosphoribosyl)-5- [(5-phosphoribosylamino) methylideneamino] imidazole-4-carboxamide isomerase APG10 (TraesCS1A01G251000.1) was the core protein in this PPI network, and it interacted with peptide chain release factor APG3

(TraesCS1B01G098300.2), cytosolic glutamine synthetase GSR2 (TraesCS4A01G266900.1),

PAL1, ADT6 and ribose-phosphate pyrophosphokinase 1 AT2G35390

(TraesCS6A01G088200.2). Flavor 3'-O-methylransferase 1 OMT1 (TraesCS4B01G352400.1)

catalyzes the methylation of lignin precursor monoglyceride and also participates in the biosynthesis of melatonin. It interacted with AT4G37520, cytochrome b-c1 complex subunit rieske, mitochondrial isoform X1 AT5G14130 (TraesCS7A01G262000.1), peroxidase 11

AT1G68850 (TraesCS7A01G211200.1), peroxidase 5-like AT1G14550

(TraesCS1B01G096900.1), peroxidase 31-like AT3G28200 (TraesCS1B01G220600.1) and

PAL1. RPL24 (TraesCS7A01G450600.1) plays a key role in the biosynthesis of thylakoid 
272 membrane proteins encoded by chloroplast genes, and it interacted with 60S ribosomal protein

273 L18-2-like RPL18 (TraesCS1D01G105500.1), nascent polypeptide-associated complex subunit

274 alpha-like protein 2 NACA2 (TraesCS4B01G347400.1), APG3 and spermidine synthase SPDS1

275 (TraesCS7B01G232700.1). OMT1 and RPL24 were the second most important hub proteins in

276 this PPI network.

277

278

279

280

281

282

283

284

285

286

287

\section{Proteome-transcriptome-associated analysis}

Using a proteome-transcriptome-associated analysis, 'response to stimulus' (GO:0009611), 'antioxidant activity', 'carbohydrate metabolism', 'amino acid metabolism', 'signal transduction', 'transport and catabolism' and 'biosynthesis of other secondary metabolites' were identified under both salt-stress and recovery conditions in 'Y1805' (Table 1). In addition, 'energy metabolism' and 'lipid metabolism' were recovery-specific pathways. In total, 13 and 25 upregulated DEPs/DEGs were identified under salt-stress and recovery conditions in 'Y1805', respectively (Table S7). Furthermore, seven DEPs/DEGs appeared under both conditions, and were probable carboxylesterase 15 CXE15 (TraesCS1D01G256800.1), abscisic acid (ABA)inducible protein PHV A1-like HVA1 (TraesCS1D01G369800.1), ADT6, PAL1 and three uncharacterized proteins (TraesCS1A01G295800.1, TraesCS1B01G304800.1 and TraesCS2D01G417100.2) (Table S7). In addition to the common proteins, there were 6 and 18 'Y1805'-specific DEPs/DEGs identified under salt-stress and recovery conditions, respectively (Table S7).

Compared with 'CS', four 'Y1805'-specific DEPs (AT1G14550, AT4G33420, AT4G37520, 
293 EMT04243) were found under salt-stress conditions. Among them, the antioxidant protein

294 AT1G14550 and molecular functional regulatory protein EMT04243 displayed the same

295

296

297

298

299

300

301

302

303

304

305

306

307

308

309

310

311

312

313

07

expression pattern at the transcriptional and proteome levels. Putative calcium-binding protein

CaM-like (CML) 16 (TraesCS3B01G045500.1) was upregulated during the recovery process

only in 'Y1805' at both the transcriptome and proteome levels (Table S7).

The functional association diagram of ' $Y 1805$ ' DEPs/DEGs under salt-stress conditions is

shown in Fig. 6. Among the associated proteins, galactinol-sucrose galactosyltransferase RFS1

(TraesCS3A01G092800.1) was upregulated in 'galactose metabolism' (ko00052) and is a key

protein in raffinose synthesis. PAL1 and AT1G14550, which are involved in the synthesis of

cinnamic acid and lignins, respectively, were upregulated in 'phenylpropanoid biosynthesis'

(ko00360). In addition, PAL1 is also a key protein in 'phenylalanine metabolism'. ADT6, which

takes part in phenylalanine synthesis, was upregulated in 'phenylalanine, tyrosine and tryptophan

biosynthesis' (ko00400).

Probable serine acetyltransferase 1 SAT1 (TraesCS3A01G287400.1) and 1-

aminocyclopropane-1-carboxylate oxidase 1 EFE (TraesCS5A01G234300.1) were upregulated

in 'cysteine and methionine metabolism' (ko00270) during the recovery process in 'Y1805' (Fig.

7). They participate in the synthesis of acetyl-L-serine and ethylene, respectively. Xylanase

inhibitor Xip-R1 (TraesCS4A01G173800.1), which is involved in the synthesis of chitobiose and

GlcNAc, was upregulated in 'amino sugar and nucleotide sugar metabolism' (ko00520). PAL1,

AT1G68850 and berberine bridge enzyme-like 27 AT5G44410 (TraesCS7B01G273600.1) were

PeerJ reviewing PDF | (2021:05:60891:2:0:NEW 31 Oct 2021) 
314 upregulated in 'phenylpropanoid biosynthesis'.

\section{DISCUSSION}

317 Plants, being sessile, have evolved specific and complex mechanisms in response to different

318 abiotic stresses at the transcriptome, proteome and physiological levels. The responses of salttolerant common wheat 'Jing411' and salt-sensitive wheat 'CS' seedling roots to salt stress have been investigated using a proteomics analysis and 52 and 47 specific DEPs were detected, respectively (Guo et al., 2012). In total, 38 DEPs have been identified under salt-stress conditions in durum wheat (Caruso et al., 2008). In this study, the DEP quantitative analysis was carried out under salt-stress and recovery conditions in 'Y1805' and 'CS'. In total, 44 and 69

DEPs were identified in 'Y1805' and 'CS', respectively, among which 21 proteins with the same

expression patterns were common to both genotypes. They represented the core genes associated with responses to salt stress. 'Y1805' had 23 specific DEPs under salt-stress conditions that

should play key roles in its strong salt tolerance. Probable ATP-dependent DNA helicase CHR12 identified under salt-stress conditions in 'Y1805' when compared with salt-tolerant common wheat (Guo et al., 2012), durum wheat (Caruso et al., 2008) and barley (Mostek et al., 2015).

ATCHR12 plays an important role in the regulation of stress-induced transient growth arrest in 
334

335

336

337

338

339

340

341

342

343

344

345

346

347

348

349

350

351

352

353

overcome or temporarily cope with stress. Here, CHR12 was 'Y1805'-specific under salt-stress conditions, during which the expression level was very high (4.0572), but not after recovery.

After recovery, 73 'Y1805'-specific DEPs were identified that should contribute to its fast recovery after removing the salt stress. In total, 14 DEPs were identified under both salt-stress and recovery conditions in 'Y1805'. More specific DEPs were identified after recovery than during the salt stress in 'Y1805'. Therefore, the responses to salt stress and recovery were two independent, but closely related, processes.

\section{Protein responses to salt stress}

\section{Antioxidant proteins}

As members of the defense system, plant antioxidant proteins remove ROS and reduce the damage caused by oxidative stress. The overexpression of class III peroxidase genes enhances the antioxidant capacity of maize under abiotic stress conditions (Wang et al., 2015). POD (B4G0X5) was upregulated in both salt-tolerant and salt-sensitive maize to improve the salt tolerance of the two maize varieties (Chen et al., 2019). Here, the 'antioxidant activity' pathway was enriched, and three peroxide superfamily proteins (AT1G14550, AT4G33420 and AT4G37520) were identified under salt-stress conditions only in 'Y1805'. AT1G14550, which could remove excessive $\mathrm{H}_{2} \mathrm{O}_{2}$, thereby enhancing the antioxidant capacity, was upregulated at both the transcriptional and protein levels. However, no POD was found in 'CS', resulting in excessive ROS accumulation, which led to oxidative damage.

\section{Osmoregulation}


354 Soluble sugars participate in osmotic regulation and also play important roles in maintaining

355 protein stability. Glycosyltransferase (A0a1d6ewj3) was upregulated in both salt-tolerant and -

356 sensitive maize cultivars, and plays an active role in salt tolerance (Chen et al., 2019). RFS1 is

357 involved in the synthesis of cottonseed sugar, which acts as an osmotic protective agent in plant

358 cells (Nishizawa, Yabuta \& Shigeoka, 2008). In this work, 'carbohydrate metabolism' was

359 significantly enriched. Trehalose has an important physiological role in osmotic protection, and it

360 improves the tolerance of plants to abiotic stress (Wang et al., 2020). In this experiment,

361 upregulated trehalose-phosphate phosphatase 3 TPP3 (TraesCS2A01G161200.1) was detected

362 only in 'Y1805', and it could remove phosphate from trehalose-6-phosphate to produce free

363 trehalose. Therefore, soluble sugars might contribute to the strong salt tolerance of 'Y1805'.

364 Phytohormones

365 ABA plays an important role in regulating water balance, osmotic protection and cell wall

366 component synthesis, and it is also involved in the control of phosphatidyl inositol signaling and

367 chromatin-mediated stress tolerance mechanisms, which alleviate the physiological effects of salt

368 stress and enhance the protective mechanisms against stress (Szypulska, Jankowski \& Weidner,

369 2017). Low doses of ABA (10-100 $\mu \mathrm{M})$ induce antioxidant defense mechanisms to prevent

370 oxidative damage to membrane lipids and proteins (Jiang \& Zhang, 2001; Wang, Kuang \& He,

371 2010). Pathogenic-associated protein 10 is involved in ABA synthesis, which is upregulated in

372 salt-tolerant maize '8723' but stable in salt-sensitive maize 'P138' (Chen et al., 2019). A similar

373 result was obtained in our study. HVA1 was upregulated at both the transcriptional and protein

374 levels under salt-stress conditions only in 'Y1805'. The upregulated expression of HVA1 
375

376

377

378

379

380

381

382

383

384

385

386

387

produces $\mathrm{ABA}$, which is beneficial to maintaining the water balance and preventing oxidative damage.

Ethylene induces the occurrence of adventitious roots and root hairs. The presence of root hairs increases the absorption area of roots, and they secrete a variety of substances, such as organic acids. Moreover, ethylene increases the flavonol content of guard cells. Flavonols act as antioxidants, reduce ROS levels in guard cells and inhibit stomatal closure (Murata, Mori \& Munemasa, 2015). An increase in 1-aminocyclopropane-1-carboxylic acid oxidase leads to a large amount of ethylene production (Qin et al., 2007). An ethylene receptor (a negative regulator of ethylene signaling) expression level increased more than nine-fold in salt-sensitive wheat 'Jinan 177' and only two-fold in salt-tolerant 'Shanrong No. 3 ' in response to salt stress. Therefore, the lower ethylene receptor expression in 'Shanrong No. 3' under salt-stress conditions may be partly responsible for its lower sensitivity to salinity than 'Jinan 177' (O'Malley et al., 2005; Wang et al., 2010). Here, upregulated EFE was found only in 'Y1805' under salt-stress conditions and is beneficial for its strong salt tolerance. Under salt-stress conditions, upregulated HVA1 and EFE play vital roles in the salt tolerance of 'Y1805'.

\section{Protein responses to recovery}

\section{Cell wall synthesis}

UDP-glucose dehydrogenase (UGD) plays a key role in the nucleotide sugar biosynthetic pathway, because its product, UDP-glucuronic acid, is the common precursor for arabinose, xylose, galacturonic acid and apiose residues found in cell walls, which play important roles in primary cell-wall formation (Reboul et al., 2011; Siddique et al., 2012). Here, an upregulated 
396

397

398

399

400

401

402

403

404

405

406

407

408

409

410

411

412

413

414

415

416

UDP-glucose 6-dehydrogenase 2, UGD2 (TraesCS4A01G237900.1), which improves the synthesis of cell-wall polymers, was detected during the recovery process only in 'Y1805'.

Caffeic acid 3-O-methyltransferase (OMT) catalyzes the multi-step methylation reactions of hydroxylated monomeric lignin precursors, which have important roles in lignin biosynthesis (Ma \& Xu, 2008). OMT1 was a 'Y1805'-specific upregulated protein, and it is involved in lignin biosynthesis. With the increase in lignin synthesis, 'Y1805' was able to repair cell walls rapidly during the recovery process, resulting in a rapid return to normal growth. These upregulated UGD2 and OMT1 proteins in 'Y1805' strengthen cell walls and play master roles in the line growth recovery.

\section{Improved respiration}

Stress conditions stimulate the expression of GPDHC1, which is related to the availability of oxygen and a key protein affecting mitochondrial respiration (Shen et al., 2006). In this study, GPDHC1 (TraesCS3A01G330600.1) was upregulated only in 'Y1805' during the recovery process, indicating that its respiration was improved, which would provide essential energy and intermediate metabolites for the plant's rapid recovery after being subjected to salt stress.

\section{Phytohormones and signal transduction}

During the recovery process, HVA1 was upregulated in both 'Y1805' and 'CS', but the expression level in 'Y1805' was higher than that in 'CS' (Table S6). OMT1 catalyzes the transfer of a methyl group onto $\mathrm{N}$-acetylserotonin, which produces melatonin $(\mathrm{N}$-acetyl-5methoxytryptamine) (Byeon et al., 2015). Melatonin has antioxidative effects in plants. It scavenges ROS mainly by providing electrons. Melatonin also promotes the formation of lateral 
417 roots. Indole-3-acetic acid (IAA)-amino acid hydrolase ILR1-like 2 produces free IAA by

418 hydrolyzing IAA-amido (Leclere et al., 2002; Rampey et al., 2004). This enzyme

419 (TraesCS3B01G283900.1) was upregulated only in 'Y1805', resulting in the production of free

420 IAA, which promotes plant growth. Salicylic acid (SA) reduces water loss by inhibiting stomatal

421 opening to alleviate salt stress. EPS1 (a BAHD acyltransferase-family protein) is an essential

422 protein for pathogen-induced SA accumulation (Torrens-Spence et al., 2019). Here, EPS1

423 (TraesCS3A01G367900.1) was upregulated only in 'Y1805', which resulted in an SA

424 accumulation. Together, these phytohormones had a critical impact on the rapid growth recovery 425 of 'Y1805'.

426 The responses of plants to abiotic stress are to consolidate their growth and development by 427 regulating complex signaling networks for stress adaptation (Abreu et al., 2013). In plants, $\mathrm{Ca}^{2+}$ 428 signals play important roles in response to abiotic stresses, such as salinity, drought and 429 temperature. The $\mathrm{CML}$ proteins $\left(\mathrm{Ca}^{2+}\right.$ sensors $)$ play unique roles in plant $\mathrm{Ca}^{2+}$-signaling 430 pathways (Ogunrinde et al., 2017). The $\mathrm{Ca}^{2+}$ signaling network is closely related to the activation of the salt overly sensitive 'signal transduction' pathway that regulates cellular $\mathrm{Na}^{+}$and $\mathrm{K}^{+}$ homeostasis in Arabidopsis (Zhu, 2002; Brini et al., 2007). In this study, the 'signal transduction' pathway was significantly enriched, and CML16 was upregulated only in 'Y1805' after recovery.

434 Therefore, $\mathrm{Ca}^{2+}$ signaling was involved in the responses to recovery in ' $\mathrm{Y} 1805$ '.

435 Transcriptional regulation and error information processing

DExH-box ATP-dependent RNA helicase DExH10 (HEN2) assists the exosome-mediated 
438

\section{DEP interactions}

457 Cell wall integrity is an important factor that determines salt-stress tolerance and plant growth

precursors and long non-coding RNAs, as well as a large number of spurious transcripts derived from antisense and non-annotated regions. In addition, HEN2 is involved in the degradation of excised introns and incompletely spliced or otherwise mis-transcribed or mis-processed mRNAs (Lange et al., 2014). DEAD-box ATP-dependent RNA helicase 14 (DRH1) is a novel type of ATP/dATP-dependent RNA helicase and polynucleotide-dependent ATPase that is capable of unwinding double-stranded RNA in the presence of ATP or dATP and of hydrolyzing ATP (Okanami, Meshi \& Iwabuchi, 1998). In this study, 'transcription regulation activity' pathway was enriched, and upregulated HEN2 (TraesCS2A01G154400.1) and DRH1 (TraesCS3A01G130200.1) were detected only in 'Y1805' during the recovery process, indicating that transcriptional regulation and error information processing performed crucial roles in the line rapid recovery from salt stress.

The wheat DEPs were enriched in 'carbon metabolism', 'detoxification', 'defense', 'signal transduction' and 'companionship' under salt-stress conditions as determined by a proteomics analysis (Caruso et al., 2008; Wang et al., 2008; Guo et al., 2012; Ma et al., 2020). Here, 'carbohydrate metabolism', 'antioxidant activity' and ‘environmental adaptation' and 'signal transduction' were also enriched in 'Y1805'. What's more, the salt tolerance of 'Y1805' might be attributed to cell-wall strengthening, transient growth arrest, osmoregulation and ABA regulation. (Feng et al., 2018; Zhao et al., 2018). Lignin is produced and the cell wall is hardened through 
459

460

461

462

463

464

465

466

467

468

469

470

471

472

473

474

475

476

477

478

479

the actions of ADT6 and PAL1 (Huang et al., 2010). CEAS1 is involved in the formation of the

primary cell wall. IRX1 participates in the formation of the secondary cell wall and plays an important role in the thickening of the xylem cell wall (Turner \& Somerville, 1997). CSLA01 is involved in the synthesis of galactomannan, which is a non-cellulose polysaccharide in plant cell walls. ADT6, PAL1, CEAS1, IRX1 and CSLA01 interacted with each other under salt-stress conditions in 'Y1805', and they were closely related to the synthesis and strength of the cell wall. In addition, IRX1 and CSLA01 were 'Y1805'-specific DEGs. Therefore, the cell wall performed major roles in the salt tolerance of 'Y1805'.

Abiotic stresses, such as salinity, drought, osmotic, cold and freezing temperatures produce cellular water deficits, which lead to the accumulation of highly hydrophilic LEA proteins (Battaglia et al., 2008; Hundertmark \& Hincha, 2018). 9 LEA DEGs (Tel3E01G270600, Tel3E01G426400, Tel3E01G628300, Tel4E01G475800, Tel5E01G589800, Tel5E01G665500,

\section{Tel6E01G622800, Tel6E01G623000 and Tel7E01G989400) originating from Thinopyrum}

elongatum were detected among top 30 DEGs under salt-stress conditions in 'Y1805' according to our transcriptome analysis. Histidine is a key component of LEA proteins. APG10 is not only a hydrophilic protein, but also an important protein in histidine biosynthesis. In this study, APG10 was the hub protein during the recovery process in 'Y1805', and it was downregulated.

The result revealed that LEA proteins contributed to salt tolerance, and their expression levels fell rapidly during the recovery process. OMT1 is involved in the biosynthesis of lignin, which is used to repair the cell wall rapidly during the recovery process in 'Y1805'. It also produced melatonin, which improved the rapid growth recovery of 'Y1805'. RPL24 is an important 
480

481

482

483

484

485

486

487

488

489

490

491

492

493

494

495

496

497

498

499

500

protein for photosynthesis and optimal plastid performance, and it plays a key role in the

biosynthesis of thylakoid membrane proteins encoded by chloroplast genes (Romani et al., 2012).

OMT1 and RPL24 were the second most important hub proteins in the 'Y1805' PPI network.

They were both 'Y1805'-specific and upregulated during the recovery process.

\section{CONCLUSION}

44 and 102 DEPs were identified in 'Y1805' under salt-stress and recovery conditions,

respectively, using the DIA proteomics techniques. In total, 13 and 25 upregulated DEPs/DEGs

were found under salt-stress and recovery conditions, respectively, using a proteome-

transcriptome-associated analysis. Compared with 'CS', 'Y1805' had specific responses to salt

stress and recovery. Scavenging ROS, osmoregulation, phytohormone regulation, transient

growth arrest and cell-wall strengthening might be of paramount importance in its strong salt

tolerance (Fig. 8). After recovery, 'Y1805' restored growth by cell-wall strengthening,

respiration enhancement, phytohormone regulation, signal transduction, and transcriptional

regulation and error information processing (Fig. 8). This work provides a new perspective on

the molecular mechanisms underlying the adaptation of Tritipyrum to salt stress, and the results

may accelerate salt-tolerant crop breeding programs.

\section{ADDITIONAL INFORMATION AND DECLARATIONS}

\section{Acknowledgments}

Special thanks are due to Pr. Adam J Lukaszewski (University of California Riverside, USA) for the technical assistance in the experiments. We thank International Science Editing 
501 (http://www.internationalscienceediting.com) for editing this manuscript.

502

503

504

505

506

507

508

509

510

511

512

513

514

515

516

517

518

519

520

521

522

523

524

525

526

527

\section{Author Contributions}

- Rui Yang, Zhifen Yang, Ze Peng, Luxi Shi and Yabing Dong performed the experiments, prepared figures and/or tables, and approved the final draft.

- Mingjian Ren and Qingqin Zhang conceived and designed the experiments, authored or reviewed drafts of the paper, and approved the final draft.

- Guangdong Geng, Suqin Zhang and Fang He conceived and designed the experiments, analyzed the data, prepared figures and/or tables, and approved the final draft.

\section{REFERENCES}

Abreu IA, Farinha AP, Negrão S, Gonçalves N, Fonseca C, Rodrigues M, Batista R, Saibo NJM, Oliveira MM. 2013. Coping with abiotic stress: Proteome changes for crop improvement. Journal of Proteomics 93: 145-168 DOI 10.1016/j.jprot.2013.07.014.

Achard P, Cheng H, De GrauweL, Decat J, Schoutteten H, Moritz T, Van Der StraetenD, Peng J, Harberd NP. 2006. Integration of plant responses to environmentally activated phytohormonal signals. Science 311: 91-94 DOI 1126/science.1118642.

Battaglia M, Olvera Carrillo Y, Garciarrubio A, Campos F, Covarrubias AA. 2008. The enigmatic LEA proteins and other hydrophilins. Plant Physiology 148: 6-24 DOI 10.1104/pp.108. 120725.

Bernhardt OM, Selevsek N, Gillet LC, Rinner O, Picotti P, Aebersold R, Reiter L. 2014. Spectronaut: a fast and efficient algorithm for MRM-like processing of data independent acquisition (SWATH-MS) data. 60th American Society for Mass Spectometry Conference.

Brini F, Hanin M, Mezghani I, Berkowitz GA, Masmoudi K. 2007. Overexpression of wheat $\mathrm{Na}^{+} / \mathrm{H}^{+}$antiporter $T N H X 1$ and $\mathrm{H}^{+}$-pyrophosphatase $T V P 1$ improve salt-and drought-stress tolerance in Arabidopsis thaliana plants. Journal of Experimental Botany 58: 301-308 DOI 10.1093/jxb/erl251.

Byeon Y, Choi GH, Lee HY, Back K. 2015. Melatonin biosynthesis requires $N$-acetylserotonin 
528

529

530

531

532

533

534

535

536

537

538

539

540

541

542

543

544

545

546

547

548

549

550

551

552

553

554

methyltransferase activity of caffeic acid $O$-methyltransferase in rice. Journal of Experimental Botany 66: 6917-6925 DOI 10.1093/jxb/erv396.

Capriotti AL, Borrelli GM, Colapicchioni V, Papa R, Piovesana S, Samperi R, Stampachiacchiere S, Laganà A. 2014. Proteomic study of a tolerant genotype of durum wheat under salt-stress conditions. Analytical and Bioanalytical Chemistry 406: 1423-1435 DOI 10.1007/s00216-013-7549-y.

Caruso G, Cavaliere C, Guarino C, Gubbiotti R, Foglia P, Laganà A. 2008. Identification of changes in Triticum durum L. leaf proteome in response to salt stress by two-dimensional electrophoresis and MALDI-TOF mass spectrometry. Analytical and Bioanalytical Chemistry 391: 381-390 DOI 10.1007/ s00216- 008-2008-x.

Chen FQ, Fang P, Peng YL, Zeng WJ, Zhao XQ, Ding YF, Zhuang ZL, Gao QH, Ren B. 2019. Comparative proteomics of salt-tolerant and salt-sensitive maize inbred lines to reveal the molecular mechanism of salt tolerance. International Journal of Molecular Sciences 20: 4725 DOI 10.3390/ijms 20194725.

Chinnusamy V, Jagendorf A, Zhu JK. 2005. Understanding and improving salt tolerance in plants. Crop Science 45: 437-448 DOI 10.2135/cropsci2005.0437.

Choi M, Chang CY, Clough T, Broudy D, Killeen D, MacLean B, Vitek O. 2014. MSstats: an R package for statistical analysis of quantitative mass spectrometry-based proteomic experiments. Bioinformatics 30: 2524-2526 DOI 10.1093/bioinformatics/btu305.

Deinlein U, Stephan AB, Horie T, Luo W, Xu G, Schroeder JI. 2014. Plant salt-tolerance mechanisms. Trends in Plant Science 19: 371-379 DOI 10.1016/j.tplants.2014.02.001.

Depuydt S, Hardtke CS. 2011. Hormone signaling crosstalk in plant growth regulation. Current Biology 21: 365-373 DOI 10.1016/j.cub.2011.03.013.

Du CX, Fan HF, Guo SR, Tezuka T, Li J. 2010. Proteomic analysis of cucumber seedling roots subjected to salt stress. Phytochemistry 71: 1450-1459 DOI 10.1016/j.phytochem.2010. 05.020.

Fahad S, Hussain S, Matloob A, Khan FA, Khaliq A, Saud S, Hassan S, Shan D, Khan F, 
555

556

557

558

559

560

561

562

563

564

565

566

567

568

569

570

571

572

573

574

575

576

577

578

579

580

581

Ullah N, Faiq M, Khan MR, Tareen AK, Khan A, Ullah A, Ullah N, Huang JL. 2015. Phytohormones and plant responses to salinity stress: a review. Journal of Plant Growth Regulation 75: 391-404 DOI 10.1007/ s10725- 014-0013-y.

Feng W, Kita D, Peaucelle A, Cartwright HN, Doan V, Duan QH, Liu MC, Maman J, Steinhorst L, Schmitz ThomI, Yvon R, Kudla J, Wu HM, Cheung AY, Dinneny JR. 2018. The FERONIA receptor kinase maintains cell-wall integrity during salt stress through $\mathrm{Ca}^{2+}$ Signaling. Current Biology 28: 666-675 DOI 10.1016/j.cub.2018.01.023.

Frukh A, Siddiqi TO, Khan MIR, Ahmad A. 2020. Modulation in growth, biochemical attributes and proteome profile of rice cultivars under salt stress. Plant Physiology and Biochemistry 146: 55-70 DOI 10.1016/j.plaphy.2019.11.011.

Ghobadi ME, Ghobadi M, Zebarjadi A. 2017. Effect of waterlogging at different growth stages on some morphological traits of wheat varieties. International Journal of Biometeorology 61: 635-645 DOI 10. 1007/s00484-016-1240-x.

Goyal E, Amit SK, Singh RS, Mahato AK, Chand S, Kanika K. 2016. Transcriptome profiling of the salt-stress response in Triticum aestivum cv. Kharchia Local. Scientific Reports 6: 27752 DOI 10.1038/srep27752.

Guo GF, Ge P, Ma CY, Li XH, Lv DW, Wang SL, Ma, WJ, Yan YM. 2012. Comparative proteomic analysis of salt response proteins in seedling roots of two wheat varieties. Journal of Proteomics 75: 1867-1885 DOI 10.1016/j.jprot.2011.12.032.

Huang JL, Gu M, Lai ZB, Fan BF, Shi K, Zhou YH, Yu JQ, Chen ZX. 2010. Functional analysis of the Arabidopsis $P A L$ gene family in plant growth, development, and response to environmental stress. Plant Physiology 153: 1526-1538 DOI 10.1104/pp.110.157370.

Hundertmark M, Hincha DK. 2008. LEA (late embryogenesis abundant) proteins and their encoding genes in Arabidopsis thaliana. BMC Genomics 9: 118 DOI 10.1186/1471-2164-9118.

Isaacson T, Damasceno CMB, Saravanan RS, He YH, Catalá C, Saladié M, Rose JKC. 2006. Sample extraction techniques for enhanced proteomic analysis of plant tissues. Nature 
582

583

584

585

586

587

588

589

590

591

592

593

594

595

596

597

598

599

600

601

602

603

604

605

606

607

608

Protocols 1: 769-774 DOI 10.1038/nprot.2006.102.

Jauhar PP. 1990. Multidisciplinary approach to genome analysis in the diploid species, Thinopyrum bessarabicum and Th. elongatum (Lophopyrum elongatum), of the triticeae. Theoretical and Applied Genetics 80: 523-536 DOI 10.1007/bf00226755.

Jiang MY, Zhang JH. 2001. Effect of abscisic acid on active oxygen species, antioxidative defence system and oxidative damage in leaves of maize seedlings. Plant and Cell Physiology 42: 1265-1273 DOI 10.1093/pcp/pce162.

King IP, Law CN, Cant KA, Orford SE, Reader SM, Miller TE. 1997. Tritipyrum, a potential new salt-tolerant cereal. Plant Breeding 116: 127-132 DOI 10.1111/j.1439-0523.1997.tb0216 6.x.

Kumar V, Khare T, Sharma M, Wani SH. 2017. ROS induced signaling and gene-expression in crops under salinity stress. In: Khan MIR (ed) Reactive oxygen species and antioxidant systems: role and regulation under abiotic stress. Springer, Singapore 159-184 DOI 10.1007/978-981-10-5254-5.

Lai Y, Zhang DQ, Wang JM, Wang JC, Ren PR, Yao LR, Si EJ, Kong YH, Wang HJ. 2020. Integrative transcriptomic and proteomic analyses of molecular mechanism responding to salt stress during seed germination in hulless barley. International Journal of Molecular Sciences 21: 359 DOI 10.3390/ijms21010359.

Lange H, Zuber H, Sement FM, Chicher J, Kuhn L, Hammann P, Brunaud V, Bérard C, Bou-teiller N, Balzergue S, Aubourg S, Martin-Magniette ML, Vaucheret H, Gagliardi D. 2014. The RNA helicases AtMTR4 and HEN2 target specific subsets of nuclear transcripts for degradation by the nuclear exosome in Arabidopsis thaliana. PLoS Genetics 10: 1004564 DOI 10.1371/ journal.pgen. 1004564 .

Leclere S, Tellez R, Rampey RA, Matsuda SPT, Bartel B. 2002. Characterization of a family of IAA-amino acid conjugate hydrolases from Arabidopsis. Journal of Biological Chemistry 277: 20446-20452 DOI 10.1074/jbc.M111955200.

Ma Q, Shi C, Su CX, Liu YG. 2020. Complementary analyses of the transcriptome and iTRAQ 
609

610

611

612

613

614

615

616

617

618

619

620

621

622

623

624

625

626

627

628

629

630

631

632

633

634

635

proteome revealed mechanism of ethylene dependent salt response in bread wheat (Triticum aestivum L.). Food Chemistry 325: 126866 DOI 10.1016/j.foodchem.2020.126866.

Ma QH, Xu Y. 2008. Characterization of a caffeic acid 3-O-methyltransferase from wheat and its function in lignin biosynthesis. Biochimie 90: 515-524 DOI 10.1016/j.biochi.2007.09. 016.

Mahajan MM, Goyal E, Singh AK, Gaikwad K, Kanika K. 2017. Transcriptome dynamics provide insights into long-term salinity stress tolerance in Triticum aestivum $\mathrm{cv}$. Kharchia Local. Plant Physiology and Biochemistry 121: 128-139 DOI 10.1016/j.plaphy.2017.10.021.

Mahajan MM, Goyal E, Singh AK, Gaikwad K, Kanika K. 2020. Shedding light on response of Triticum aestivum cv. Kharchia Local roots to long-term salinity stress through transcriptome profiling. Plant Growth Regulation 90: 369-381 DOI 10.1007/s10725-019-00 $565-4$.

Margiotta B, Colaprico G, Urbano M, Veronico G, Tommasi F, Tomaselli V. 2020. Halophile wheatgrass Thinopyrum elongatum (Host) D.R. Dewey (poaceae) in three Apulian coastal wetlands: vegetation survey and genetic diversity. Plant Biosystems 6: 1-15 DOI 10.1080/11 263504.2020.1829732.

Meng XQ, Liu SY, Dong TT, Xu T, Ma DF, Pan SY, Li ZY, Zhu MK. 2020. Comparative transcriptome and proteome analysis of salt-tolerant and salt-sensitive sweet potato and overexpression of IbNAC7 confers salt tolerance in Arabidopsis. Frontiers in Plant Science 11: 922-934 DOI 10.3389/fpls.2020.572540.

Mostek A, Börner A, Badowiec A, Weidner S. 2015. Alterations in root proteome of saltsensitive and tolerant barley lines under salt stress conditions. Journal of Plant Physiology 174: 166-176 DOI 10.10 16/j.jplph.2014.08.020.

Munns R, Tester M. 2008. Mechanisms of salinity tolerance. Annual Review of Plant Biology 59: 651-681 DOI 10.1146/annurev.arplant.59.032607.092911.

Murata Y, Mori IC, Munemasa S. 2015. Diverse stomatal signaling and the signal integration 
636

637

638

639

640

641

642

643

644

645

646

647

648

649

650

651

652

653

654

655

656

657

658

659

660

661

662

mechanism. Annual Review of Plant Biology 66: 369-392 DOI 10.1146/annurev-arplant043014-11 4707.

Nishizawa A, Yabuta Y, Shigeoka S. 2008. Galactinol and raffinose constitute a novel function to protect plants from oxidative damage. Plant Physiology 147: 1251-1263 DOI 10.1104/pp.108.122465.

O’Malley RC, Rodriguez FI, Esch JJ, Binder BM, O’Donnell P, Klee HJ, Bleecker AB. 2005. Ethylene-binding activity, gene expression levels, and receptor system output for ethylene receptor family members from Arabidopsis and tomato. Plant Journal 41: 651-659 DOI 10.1111/j.1365-313X.2004.02331.x.

Ogunrinde A, Munro K, Davidson A, Ubaid M, Snedden WA. 2017. Arabidopsis calmodulinlike proteins, CML15 and CML16 possess biochemical properties distinct from calmodulin and show non-overlapping tissue expression patterns. Frontiers in Plant Science 8: 2175 DOI 10.1111/j.136 5-313x.2004.02331.x.

Okanami M, Meshi T, Iwabuchi M. 1998. Characterization of a DEAD box ATPase/RNA helicase protein of Arabidopsis thaliana. Nucleic Acids Research 26: 2638-2643 DOI 10.1093/nar/ 26.11.2638.

Qin YM, Hu CY, Pang Y, Kastaniotis AJ, Hiltunen JK, Zhu YX. 2007. Saturated very-longchain fatty acids promote cotton fiber and Arabidopsis cell elongation by activating ethylene biosynthesis. Plant cell 19: 3692-3704 DOI 10.1105/tpc.107.054437.

Rampey RA, LeClere S, Kowalczyk M, Ljung K, Sandberg G, Bartel B. 2004. A family of auxin-conjugate hydrolases that contributes to free indole-3-acetic acid levels during Arabidopsis germination. Plant Physiology 135: 978-988 DOI 10.1104/pp.104.039677.

\section{Reboul R, Geserick C, Pabst M, Frey B, Wittmann D, Lütz-Meindl U, Léonard R,} Tenhaken R. 2011. Down-regulation of UDP glucuronic acid biosynthesis leads to swollen plant cell walls and severe developmental defects associated with changes in pectic polysaccharides. Journal of Biological Chemistry 286: 39982-39992 DOI 10.1074/jbc.m111.255695. 
663

664

665

666

667

668

669

670

671

672

673

674

675

676

677

678

679

680

681

682

683

684

685

686

687

688

689

Romani I, Tadini L, Rossi F, Masiero S, Pribil M, Jahns P, Kater M, Leister D, Pesaresi P. 2012. Versatile roles of Arabidopsis plastid ribosomal proteins in plant growth and development. Plant Journal 72: 922-934 DOI 10.1111/tpj.12000.

Setter TL, Waters I, Stefanova K, Munns R, Barrett-Lennard EG. 2016. Salt tolerance, date of flowering and rain affect the productivity of wheat and barley on rainfed saline land. Field Crops Research 194: 31-42 DOI 10.1016/j.fcr.2016.04.034.

Shen WY, Wei YD, Dauk M, Tan YF, Taylor DC, Selvaraj G, Zou JT. 2006. Involvement of a glycerol-3-phosphate dehydrogenase in modulating the $\mathrm{NADH} / \mathrm{NAD}^{+}$ratio provides evidence of a mitochondrial glycerol-3-phosphate shuttle in Arabidopsis. Plant Cell 18: 422-441 DOI 10.1105/tpc.105.039750.

Siddique S, Sobczak M, Tenhaken R, Grundler FMW, Bohlmann H. 2012. Cell wall ingrowths in nematode induced syncytia require UGD2 and UGD3. Plos one 7: 41515 DOI 10.1371/ journal.pone.0041515.

Sobhanian H, Aghaei K, Komatsu S. 2011. Changes in the plant proteome resulting from salt stress: toward the creation of salt-tolerant crops? Journal of Proteomics 74: 1323-1337 DOI 10.1016/ j. jprot.2011.03.018.

Szypulska E, Jankowski K, Weidner S. 2017. ABA pretreatment can limit salinity-induced proteome changes in growing barley sprouts. Acta Physiologiae Plantarum 39: 190 DOI 10.1007/s11738-017-2490-X.

Tang XL, Mu XM, Shao HB, Wang HY, Brestic M. 2015. Global plant-responding mechanisms to salt stress: Physiological and molecular levels and implications in biotechnology. Critical Reviews in Biotechnology 35: 425-437 DOI 10.3109/07388551.2014.889080.

Torrens-Spence MP, Bobokalonova A, Carballo V, Glinkerman CM, Pluskal T, Shen A, Weng JK. 2019. PBS3 and EPS1 complete salicylic acid biosynthesis from isochorismate in Arabidopsis. Molecular Plant 12: 1577-1586 DOI 10.1016/j.molp.2019.11.005.

Turner SR, Somerville CR. 1997. Collapsed xylem phenotype of Arabidopsis identifies 
690

691

692

693

694

695

696

697

698

699

700

701

702

703

704

705

706

707

708

709

710

711

712

713

714

715

716

717

mutants deficient in cellulose deposition in the secondary cell wall. Plant cell 9: 689-701 DOI 10.2307/3870425.

Wang K, Li FJ, Gao ML, Huang YC, Song ZG. 2020. Mechanisms of trehalose-mediated mitigation of Cd toxicity in rice seedlings. Journal of Cleaner Production 267: 121982 DOI 10.1016/j.jclepro.2020.121982.

Wang MC, Peng ZY, Li CL, Li F, Liu C, Xia GM. 2008. Proteomic analysis on a high salt tolerance introgression strain of Triticum aestivum/Thinopyrum ponticum. Proteomics 8: 1470-1489 DOI 10.1002/pmic.200700569.

Wang XQ, Kuang TY, He YK. 2010. Conservation between higher plants and the moss Physcomitrella patens in response to the phytohormone abscisic acid: a proteomics analysis. Bmc Plant Biology 10: 192 DOI doi.org/10.1186/1471-2229-10-192.

Wang Y, Wang QQ, Zhao Y, Han GM, Zhu SW. 2015. Systematic analysis of maize class III peroxidase gene family reveals a conserved subfamily involved in abiotic stress response. Gene 566: 95-108 DOI 10.1016/j.gene.2015.04.041/10.1016/j.gene.2015.04.041.

Yan MK, Xue CW, Xiong Y, Meng XX, Li BJ, Shen RF, Lan P. 2020. Proteomic dissection of the similar and different responses of wheat to drought, salinity and submergence during seed germination. Journal of Proteomics 220: 103756 DOI 10.1016/j.jprot.2020.103756.

Yuan WY, Tomita M. 2015. Thinopyrum ponticum chromatin-integrated wheat genome shows salt-tolerance at germination stage. International Journal of Molecular Sciences 16: 4512-4517 DOI 10.3390/ijms16034 512.

Zhao CZ, Zayed O, Yu ZP, Jiang W, Zhu PP, Hsu CC, Zhang LR, Tao WA, Lozano-Durán R, Zhu JK. 2018. Leucine-rich repeat extensin proteins regulate plant salt tolerance in Arabidopsis. Proceedings of the National Academy of Sciences of the United States of America 115: 13123-13128 DOI 10.1073/pnas.1816991115.

Zhao CZ, Zhang H, Song CP, Zhu JK, Shabala S. 2020. Mechanisms of plant responses and adaptation to soil salinity. Therapeutic Innovation \& Regulatory Science 1: 100017 DOI 10.1016/j.xinn.2020.100017. 
718 Zhu JK. 2001. Plant salt tolerance. Trends in Plant Science 6: 66-71 DOI 10.1016/s1360$719 \quad 1385(00) 01838-0$.

720 Zhu JK. 2002. Salt and drought stress signal transduction in plants. Annual Review of Plant 721 Biology 53: 247-273 DOI 10.1146/annurev.arplant.53.091401.143329. 


\section{Figure 1}

Figure 1 Effects of salt stress and recovery on the plant length $(A)$ and dry weight $(B)$ in two wheat varieties.

'Y1805' and 'CS' represent Tritipyrum and wheat 'Chinese Spring', respectively. T1, T2 and T3 indicate $5 \mathrm{~h}$ after exposure to salt stress, $1 \mathrm{~h}$ and $24 \mathrm{~h}$ after recovery, respectively. The treatment indicates salt stress at the T1 stage, and recovery at the T2 and T3 stages, respectively, and the control shows its respective corresponding control plant. Bars show means \pm SDs $(n=3)$. Values with different letters are significantly different at $p<0.05$.
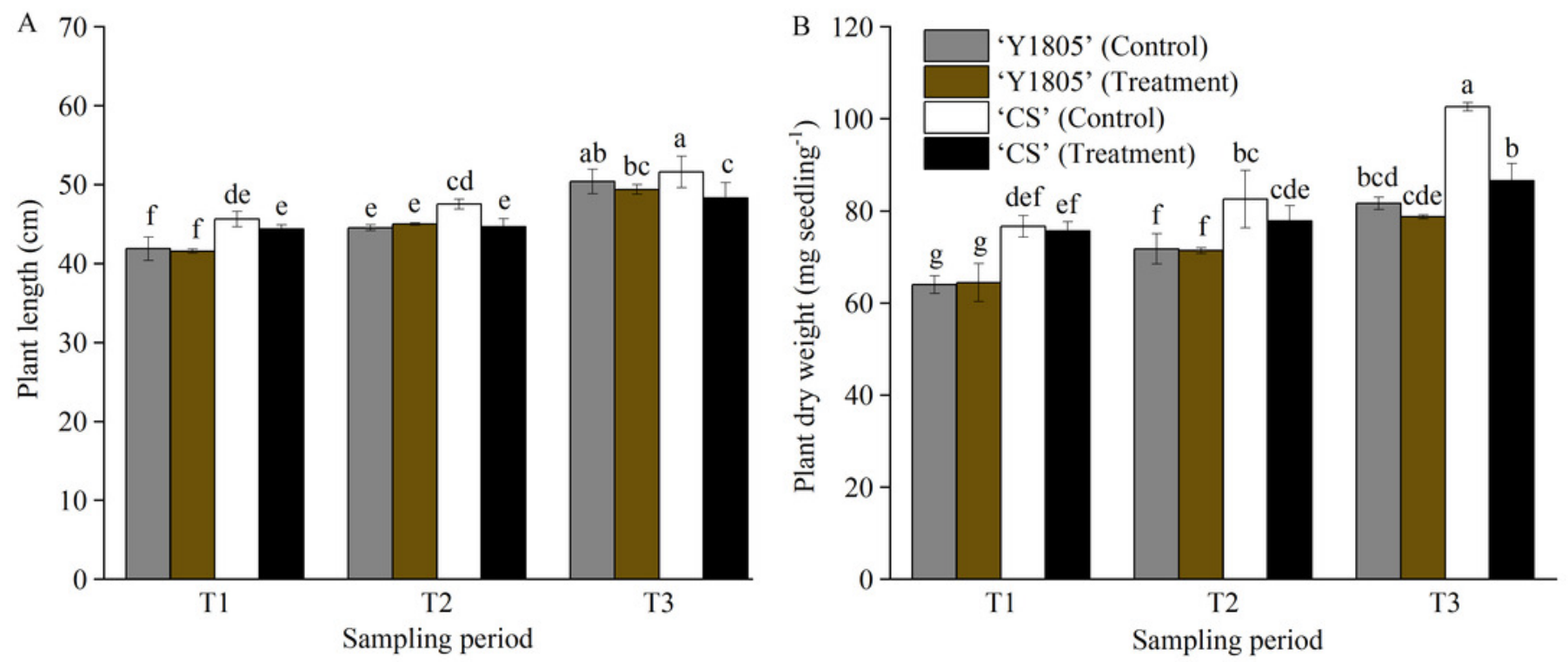


\section{Figure 2}

Figure 2 Differentially expressed protein statistics (A), Venn map (B) and qRT-PCR confirmation (C).

(A, B) CS represents wheat 'Chinese Spring'. T1 and T2 indicate salt stress and recovery, respectively. (C) The $x$ - and $y$-axes show the relative expression levels ( $\log _{2}$ fold change) analyzed independently by qRT-PCR and proteome, respectively.
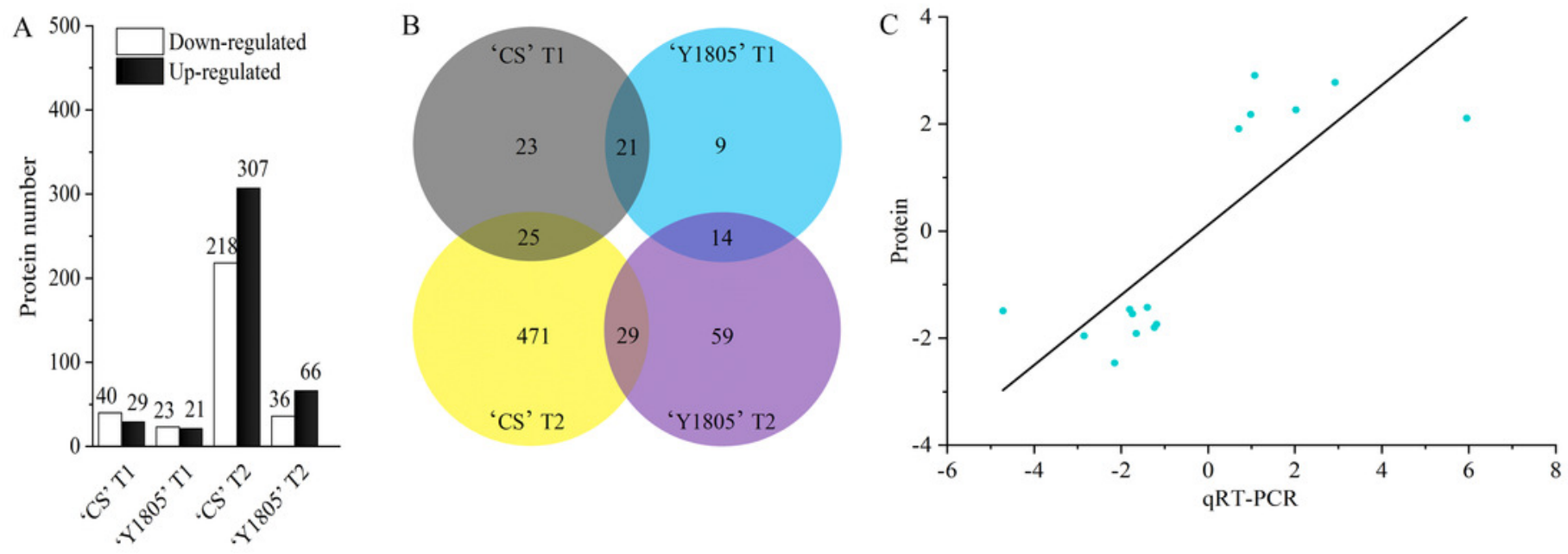
Figure 3

Figure 3 GO terms (A), KEGG pathways (B) and subcellular localization (C) of ' $Y 1805$ 'specific differentially expressed proteins under salt stress.

Cyto, chlo, extr, mito, plas and nucl indicate cytosol, chloroplast, extracellular, mitochondria, plasma membrane, and nucleus, respectively. 
A

Metabolic process

Cellular process

Cellular component organization or biogenesis

Response to stimulus
Biological regulation

Biological regulation
Cell

Cell
part

Membrane part

Membrane

Extracellular region

Organelle

Symplast

Organelle part

Cell junction

Catalytic activity Binding

Antioxidant activity

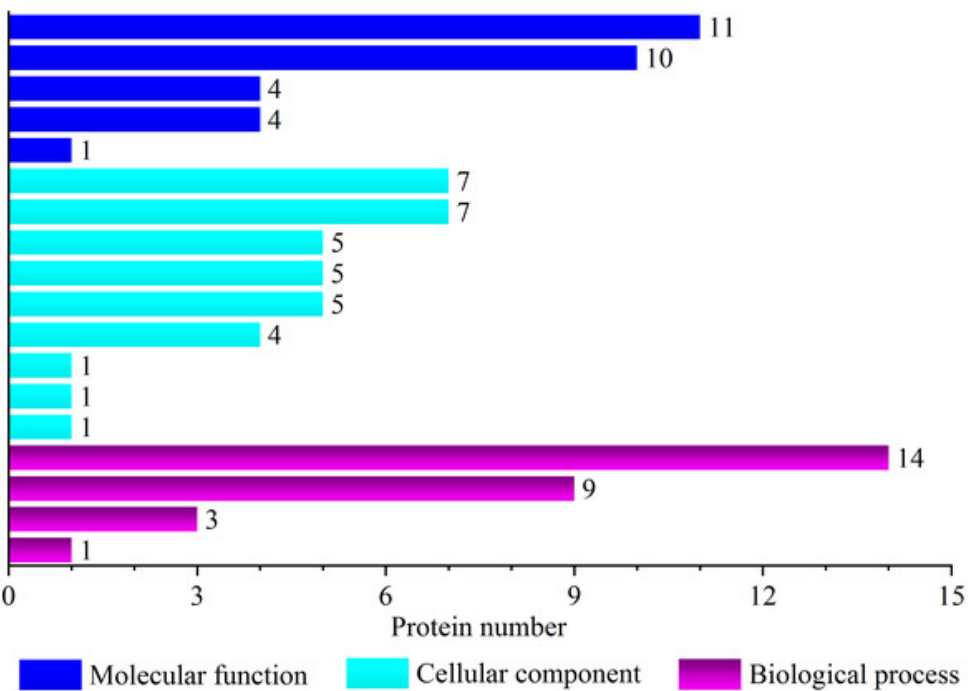

Molecular function regulator

Cellular component

Biological process

B

Transport and catabolism

Signal transduction

Translation

Folding, sorting and degradation

Global and overview maps

Carbohydrate metabolism

Biosynthesis of other secondary metabolites

Lipid metabolism

Glycan biosynthesis and metabolism

Amino acid metabolism

Metabolism of other amino acids

Metabolism of cofactors and vitamins

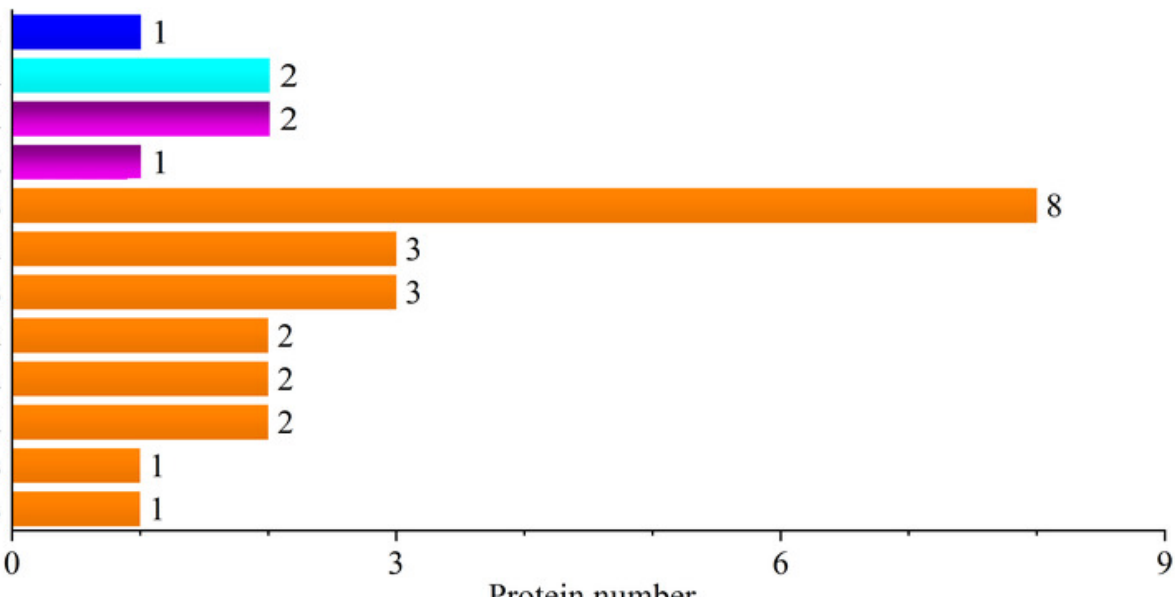

Protein number

Cellular processes

Genetic information processing

Environmental information processing

Metabolism

C

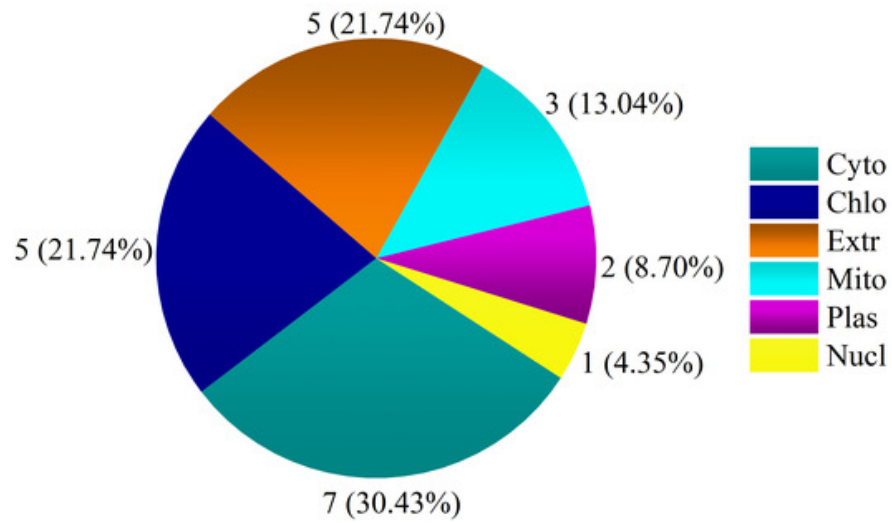




\section{Figure 4}

Figure $4 \mathrm{GO}$ terms (A), KEGG pathways (B) and subcellular localizations (C) of ' $\mathrm{Y} 1805$ 'specific differentially expressed proteins during the recovery process.

Chlo, cyto, nucl, mito, extr, plas, cysk, vacu and E.R. indicate chloroplast, cytosol, nucleus, mitochondria, extracellular, plasma membrane, cytoskeleton, vacuolar membrane and endoplasmic reticulum, respectively. 
A

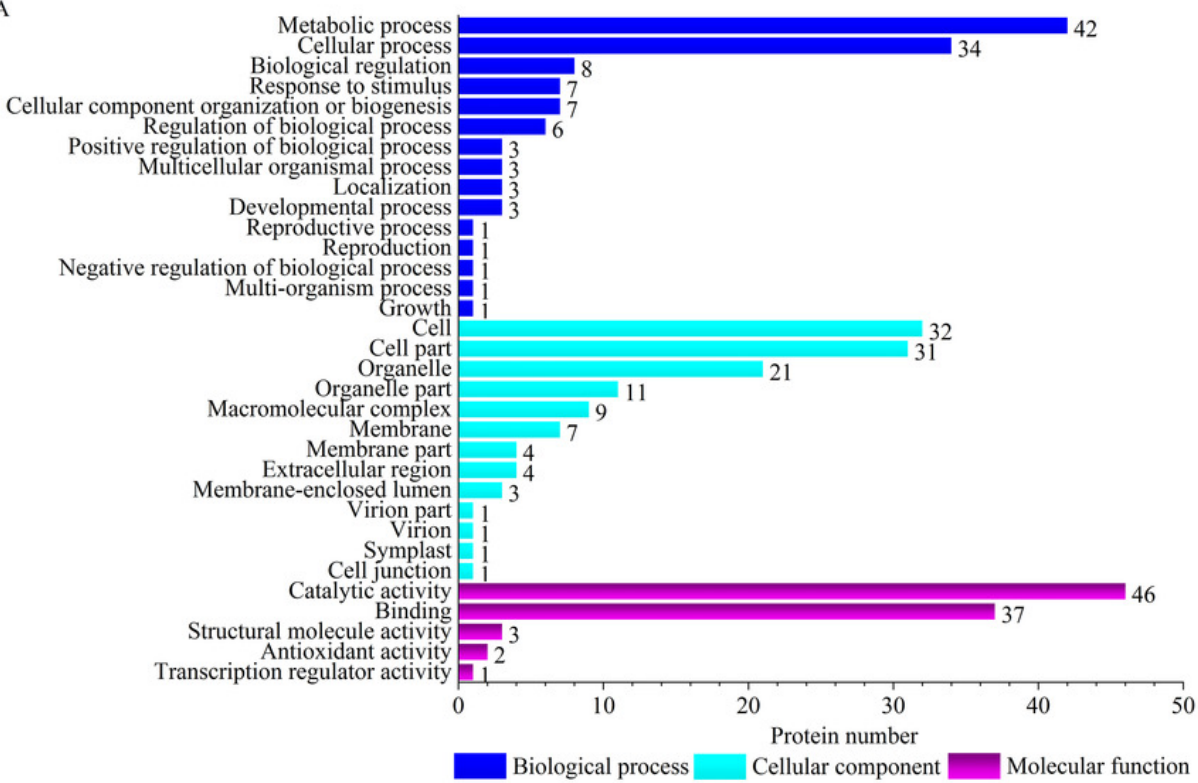

B

Transport and catabolism

Signal transduction

Membrane transport

Translation

Folding, sorting and degradation

Transcription

Replication and repair

Global and overview maps

Biosynthesis of other secondary metabolites

Carbohydrate metabolism

Amino acid metabolism

Lipid metabolism

Nucleotide metabolism

Metabolism of terpenoids and polyketides

Metabolism of cofactors and vitamins

Glycan biosynthesis and metabolism

Energy metabolism

Environmental adaptation
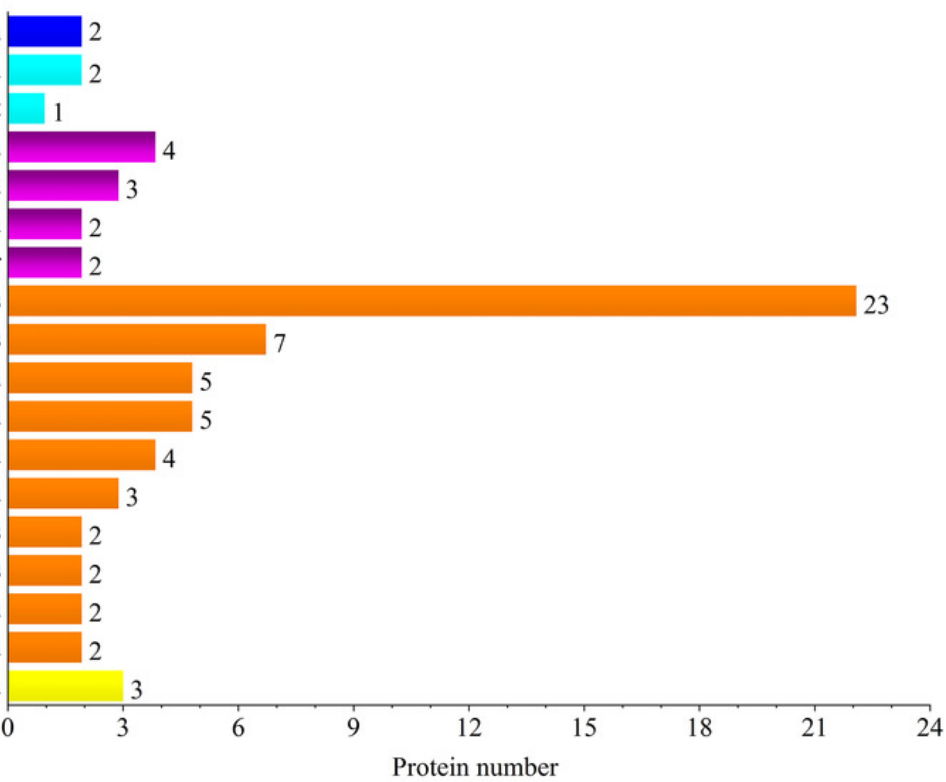

Cellular processes

Environmental information processing Genetic information processing Metabolism

Organismal systems

C

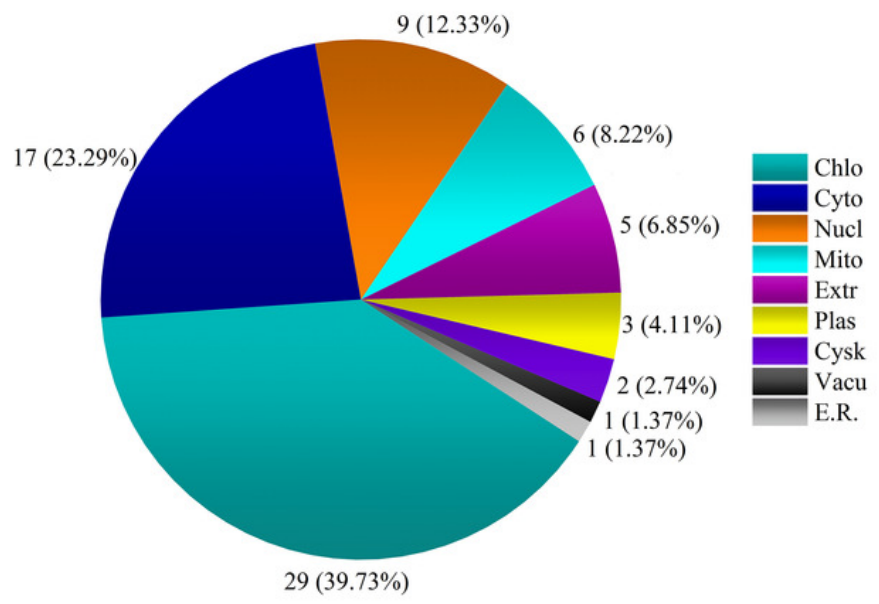




\section{Figure 5}

Figure 5 Tritipyrum ' $\mathrm{Y} 1805$ ' network diagram of protein interactions under salt-stress (A) and recovery (B) conditions.

Yellow lines represent text mining evidence, purple lines represent experimental evidence, sky blue lines represent database evidence, black lines represent co-expression evidence, green lines represent gene neighborhoods, red lines represent gene fusions and blue lines represent gene co-occurrence database evidence. PAL1, phenylalanine ammonia-lyase 1; ADT6, arogenate dehydratase/prephenate dehydratase 6; IRX1, probable cellulose synthase A catalytic subunit 8 (UDP-forming); CESA1, probable cellulose synthase A catalytic subunit 1 (UDP-forming); CSLA01, glucomannan 4-beta-mannosyltransferase 1; GH9B13, endoglucanase 17; APG3, peptide chain release factor APG3; APG10, (1-(5-phosphoribosyl)-5[(5-phosphoribosylamino) methylideneamino] imidazole-4-carboxamide isomerase; GSR2, cytosolic glutamine synthetase; AT2G35390, ribose-phosphate pyrophosphokinase 1; OMT1, flavone 3"-O-methyltransferase 1; ATG37520, peroxidase 50-like; AT5G13430, cytochrome bC1 complex subunit rieske, mitochondrial isoform X1; AT1G68850, peroxidase 11; AT1G14550, peroxidase 5-like; AT3G28200, peroxidase 31-like; RPL18, 60S ribosomal protein L18-2-like; NACA2, nascent polypeptide-associated complex subunit alpha-like protein 2; SPDS1, spermidine synthase. 
A

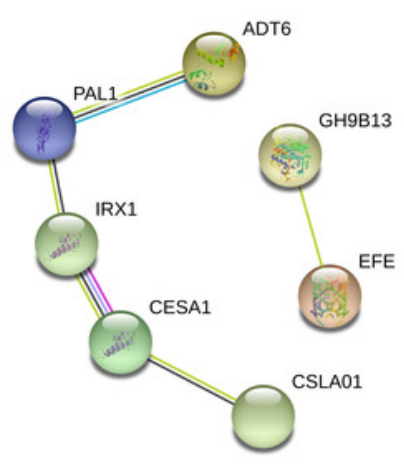

B

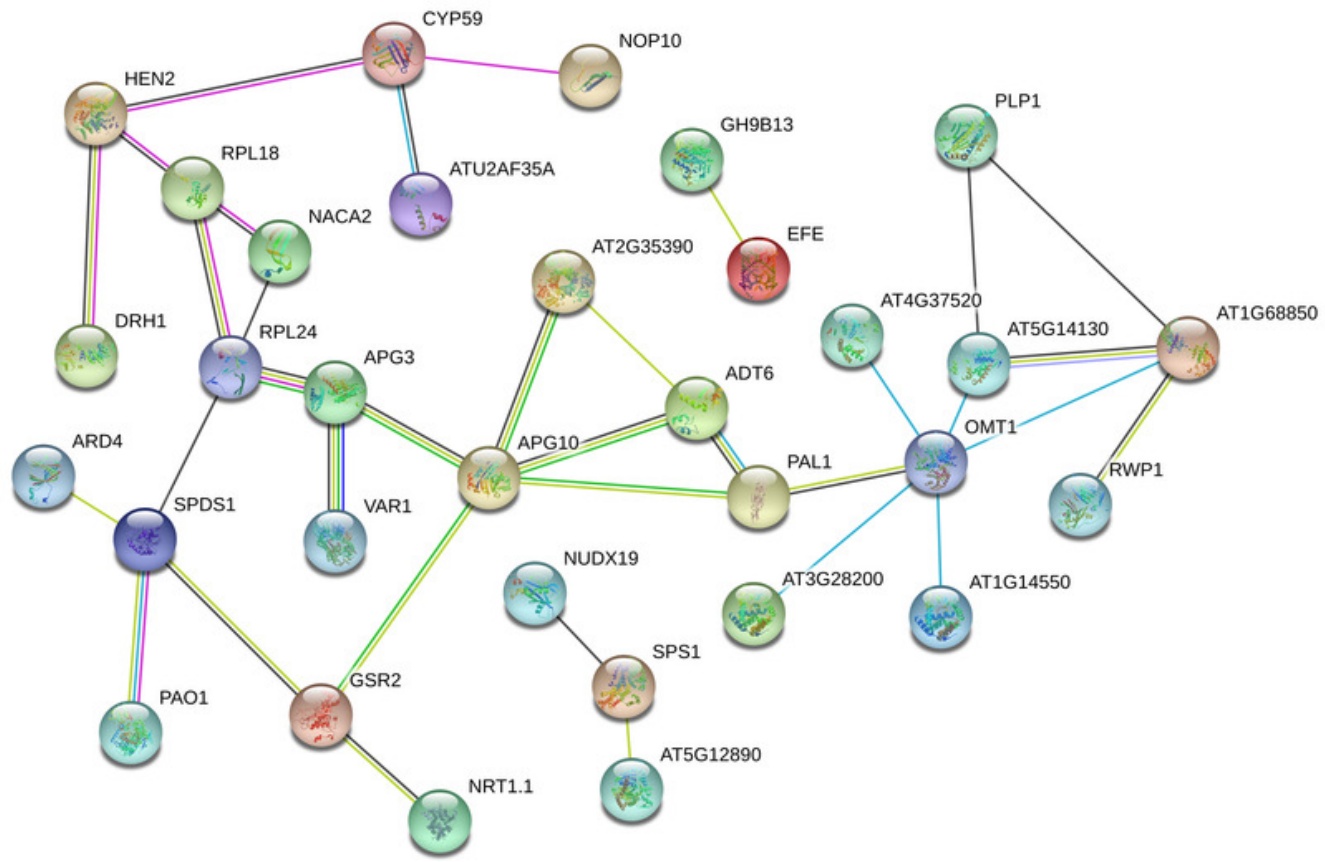


Figure 6

Figure 6 Functional association diagram of expression patterns of proteins and genes under salt-stress conditions in Tritipyrum 'Y1805'.

(A) A part of 'galactose metabolism' pathway; (B) a part of 'phenylalanine metabolism'

pathway; (C) a part of 'phenylalanine, tyrosine and tryptophan biosynthesis' pathway; (D) a part of 'phenylpropanoid biosynthesis' pathway. The blue boxes indicate that the expression patterns of the protein and gene are the same.
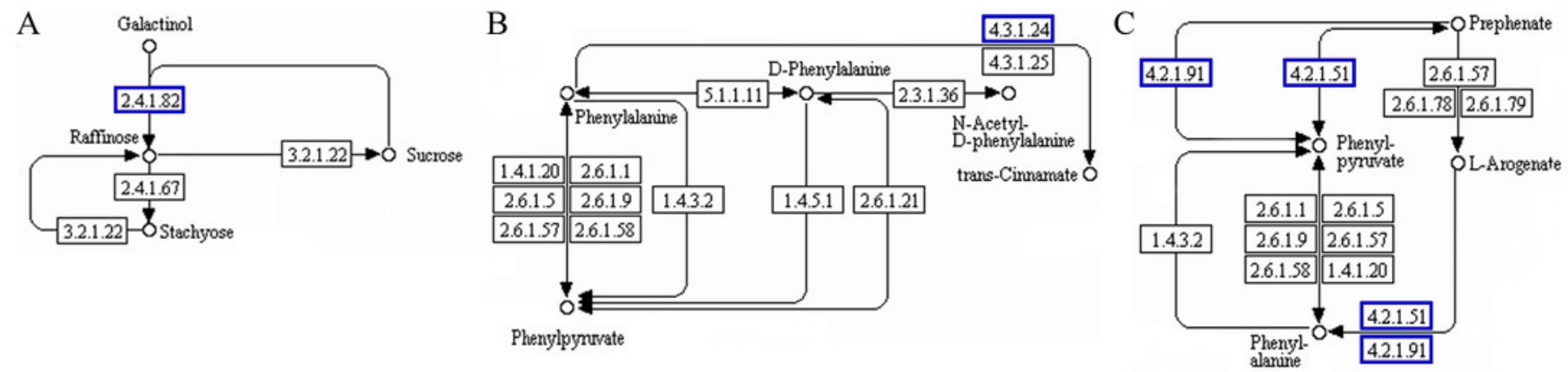

$\mathrm{D}$

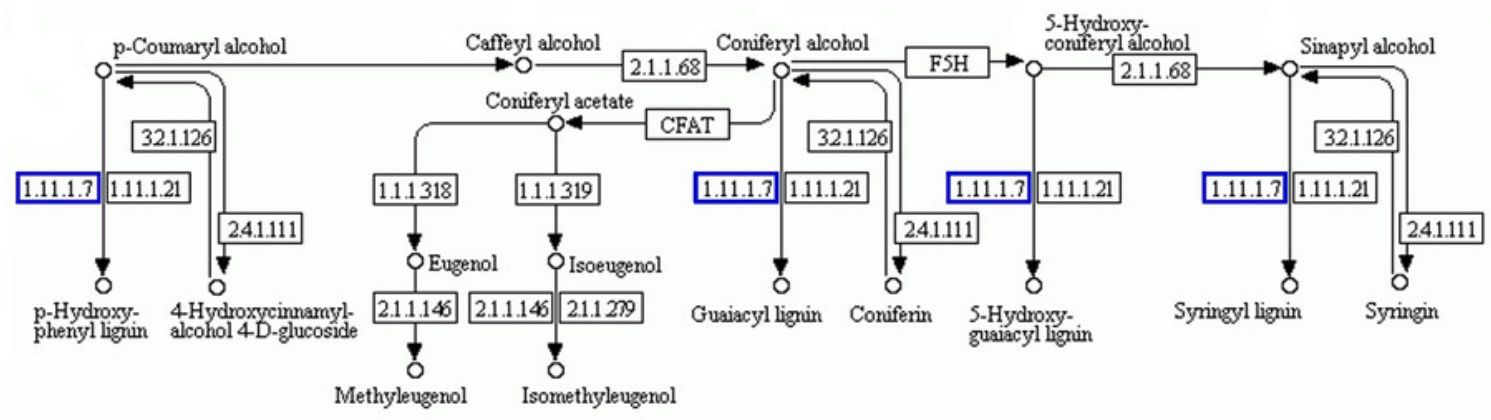




\section{Figure 7}

Figure 7 Functional association diagram of expression patterns of proteins and genes during the recovery process in Tritipyrum ' $Y 1805$ '.

(A) A part of 'cysteine and methionine metabolism' pathway; (B) a part of 'amino sugar and nucleotide sugar metabolism' pathway; (C) a part of 'phenylpropanoid biosynthesis' pathway. The blue boxes indicate that the expression patterns of the protein and gene are the same.

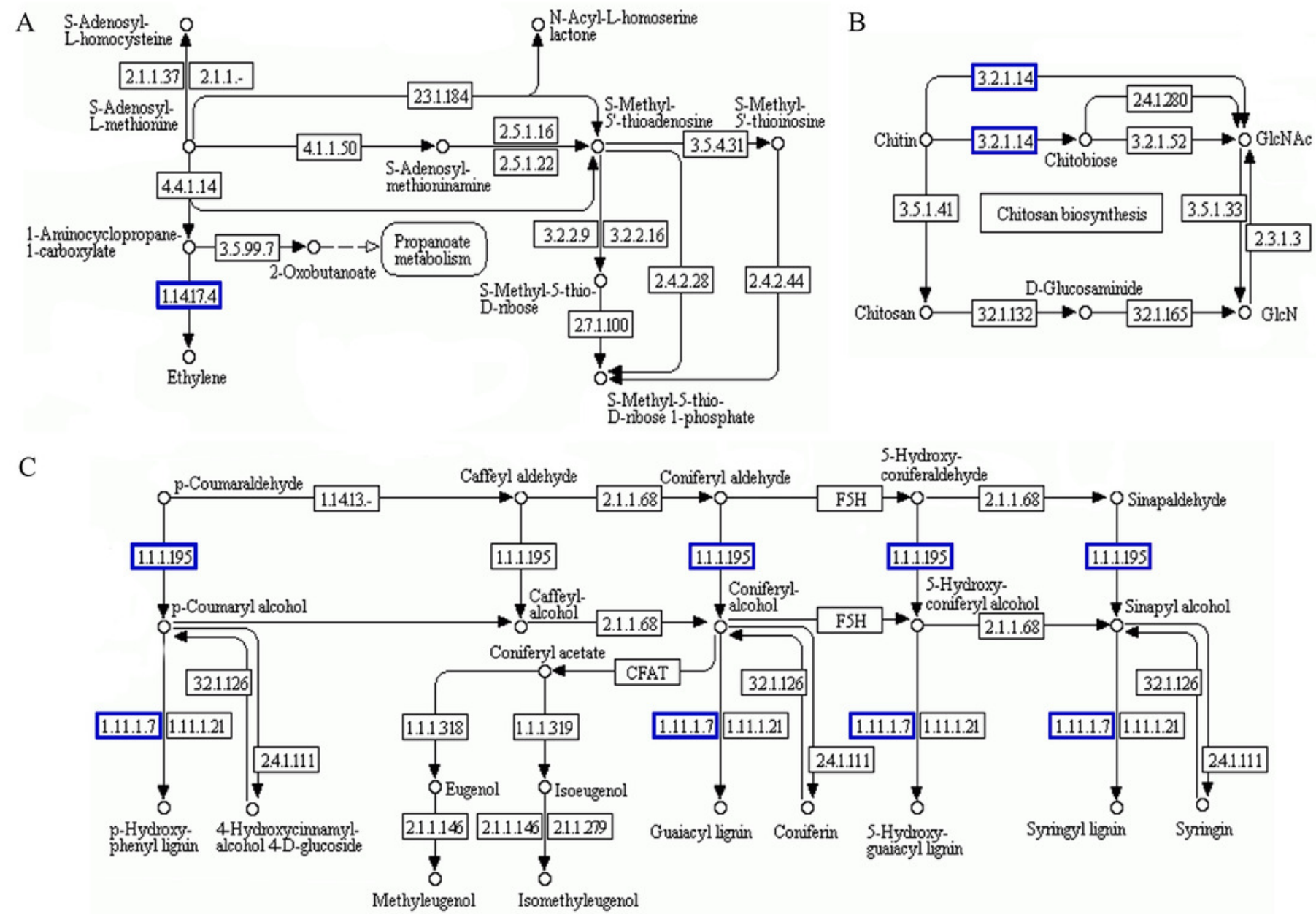




\section{Figure 8}

Figure 8 Schematic representation of the responses to salt stress and recovery in Tritipyrum 'Y1805'.

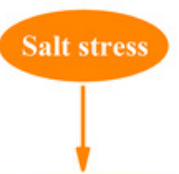

Signal transduction CML16, HVA1, EFE

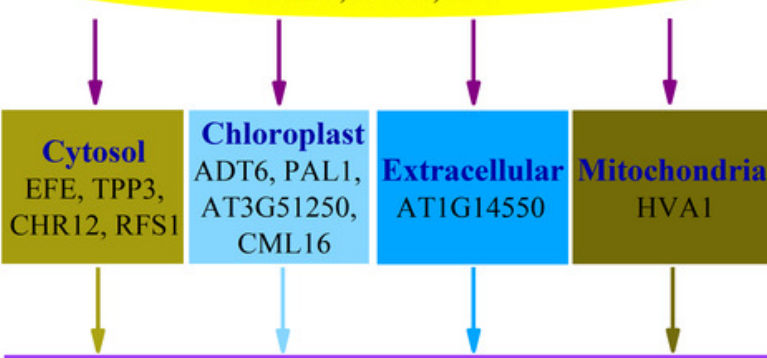

Phytohormone regulation, osmoregulation, transient growth arrest, cell wall strengthen, and ROS scavenging.

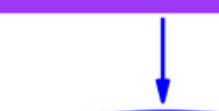

Salt tolerance

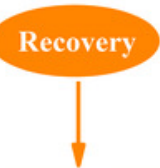

Signal transduction CML16, HVA1, EFE

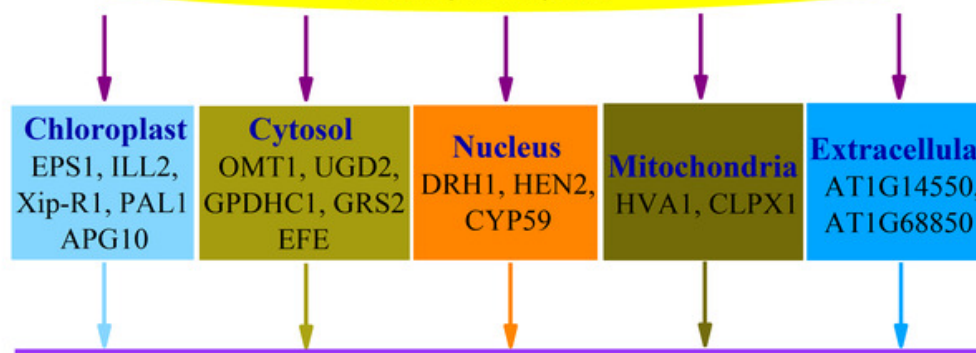

Phytohormone regulation, osmoregulation, cell wall strengthen, respiration enhancing, transcriptional regulation and error information processing, and ROS scavenging.

Y1805 adaption to salt stress 


\section{Table $\mathbf{1}$ (on next page)}

Table 1 Differentially expressed protein number, GO terms and KEGG pathways identified using a proteome-transcriptome-associated analysis under salt-stress and recovery conditions in Tritipyrum 'Y1805'. 
1 Table 1 Differentially expressed protein number, GO terms and KEGG pathways identified 2 using a proteome-transcriptome-associated analysis under salt-stress and recovery conditions in 3 Tritipyrum 'Y1805'.

\begin{tabular}{|c|c|c|c|}
\hline \multirow{2}{*}{$\begin{array}{l}\text { GO/pathway } \\
\text { ID }\end{array}$} & \multirow{2}{*}{ GO terms/pathways, level 2} & \multicolumn{2}{|c|}{ Number of DEPs/ DEGs } \\
\hline & & Salt stress & Recovery \\
\hline GO:0009611 & Response to stimulus & 3 & 1 \\
\hline GO:0004601 & Antioxidant activity & 1 & 1 \\
\hline ko04144 & Transport and catabolism & 2 & 1 \\
\hline ko04075 & Signal transduction & 1 & 2 \\
\hline ko00360 & Amino acid metabolism & 2 & 4 \\
\hline ko00940 & $\begin{array}{l}\text { Biosynthesis of other secondary } \\
\text { metabolites }\end{array}$ & 2 & 3 \\
\hline ko00052 & Carbohydrate metabolism & 1 & 3 \\
\hline ko00920 & Energy metabolism & 0 & 1 \\
\hline ko00561 & Lipid metabolism & 0 & 2 \\
\hline & & & \\
\hline & & & \\
\hline & & & \\
\hline & & & \\
\hline
\end{tabular}

